\title{
PLÜCKER FORMS AND THE THETA MAP
}

\author{
SONIA BRIVIO AND ALESSANDRO VERRA
}

\begin{abstract}
Let $S U_{X}(r, 0)$ be the moduli space of semistable vector bundles of rank $\mathrm{r}$ and trivial determinant over a smooth, irreducible, complex projective curve $X$. The theta map $\theta_{r}: S U_{X}(r, 0) \rightarrow \mathbb{P}^{N}$ is the rational map defined by the ample generator of Pic $S U_{X}(r, 0)$. The main result of the paper is that $\theta_{r}$ is generically injective if $g>>r$ and $X$ is general. This partially answers the following conjecture proposed by Beauville: $\theta_{r}$ is generically injective if $X$ is not hyperelliptic. The proof relies on the study of the injectivity of the determinant map $d_{E}: \wedge^{r} H^{0}(E) \rightarrow H^{0}(\operatorname{det} E)$, for a vector bundle $E$ on $X$, and on the reconstruction of the Grassmannian $G(r, r m)$ from a natural multilinear form associated to it, defined in the paper as the Plücker form. The method applies to other moduli spaces of vector bundles on a projective variety $X$.
\end{abstract}

\section{INTRODUCTION}

In this paper we introduce the elementary notion of Plücker form of a pair $(E, S)$, where $E$ is a vector bundle of rank $r$ on a smooth, irreducible, complex projective variety $X$ and $S \subset H^{0}(E)$ is a subspace of dimension $\mathrm{rm}$. Then we apply this notion to the study of the moduli space $S U_{X}(r, 0)$ of semistable vector bundles of rank $r$ and trivial determinant on a curve $X$. Let

$$
\theta_{r}: S U_{X}(r, 0) \rightarrow \mathbb{P}\left(H^{0}(\mathcal{L})^{*}\right)
$$

be the so called theta map, defined by the ample generator $\mathcal{L}$ of $\operatorname{Pic} S U_{X}(r, 0),[\mathrm{DN}]$. Assume $X$ has genus $g$, we prove the following main result:

Main Theorem $\theta_{r}$ is generically injective if $X$ is general and $g>>$.

The theorem gives a partial answer to the following conjecture, or optimistic speculation, proposed by Beauville in [B2] 6.1:

Speculation $\theta_{r}$ is generically injective if $X$ is not hyperelliptic.

To put in perspective our result we briefly recall some open problems on $\theta_{r}$ and some known results, see [B2]. A serious difficulty in the study of $\theta_{r}$ is represented by its indeterminacy locus, which is quite unknown. Raynaud bundles and few more constructions provide examples of points in this locus when $r>>0$, cf. [CGT] and [R]. In particular, there exists an integer $r(X)>0$ such that $\theta_{r}$ is not a morphism as soon as $r>r(X)$. As a matter of fact related to this situation, some basic questions are still unsolved. For instance:

$\circ$ is $\theta_{r}$ generically finite onto its image for any curve $X$ ?

$\circ$ is $\theta_{r}$ an embedding if $r$ is very low and $X$ is general?

- compute $r(g):=\min \{r(X), X$ curve of genus $g\}$.

On the side of known results only the case $r=2$ is well understood: $\theta_{2}$ is an embedding unless $X$ is hyperelliptic of genus $g \geq 3$, see [B1], [BV1], [vGI]. Otherwise $\theta_{2}$ is a finite 
2:1 cover of its image, [DR]. For $r=3$ it is conjectured that $\theta_{3}$ is a morphism and this is proved for $g \leq 3$, see [B2] 6.2 and [B3]. To complete the picture of known results we have to mention the case of genus two. In this case $\theta_{r}$ is generically finite, see [B3] and [BV2]. Moreover it is a morphism iff $r \leq 3,[\mathrm{~Pa}]$.

To prove our main theorem we apply a more general method, working in principle for more moduli spaces of vector bundles over a variety $X$ of arbitrary dimension. Let us briefly describe it.

Assume $X$ is embedded in $\mathbb{P}^{n}$ and consider a pair $(E, S)$ such that: (i) $E$ is a vector bundle of rank $r$ on $X$, (ii) $S$ is a subspace of dimension $r m$ of $H^{0}(E)$, (iii) det $E \cong$ $\mathcal{O}_{X}(1)$. Under suitable stability conditions there exists a coarse moduli space $\mathcal{S}$ for $(E, S)$, see for instance [L] for an account of this theory. Let $p_{i}: X^{m} \rightarrow X$ be the $i$-th projection and let

$$
e_{S, E}: S \otimes \mathcal{O}_{X^{m}} \rightarrow \bigoplus_{i=1, \ldots, m} p_{i}{ }^{*} E
$$

be the natural map induced by evaluating global sections. We will assume that $e_{E, S}$ is generically an isomorphism for general pairs $(E, S)$. For such a pair the degeneracy scheme $\mathbb{D}_{E, S}$ of $e_{E, S}$ is a divisor in $X^{m}$, moreover

$$
\mathbb{D}_{E, S} \in\left|\mathcal{O}_{X^{m}}(1, \ldots, 1)\right|,
$$

where $\mathcal{O}_{X^{m}}(1, \ldots, 1):=p_{1}^{*} \mathcal{O}_{X}(1) \otimes \cdots \otimes p_{m}^{*} \mathcal{O}_{X}(1)$. In this paper $\mathbb{D}_{E, S}$ is defined as the Plücker form of $(E, S)$. The construction of the Plücker form of $(E, S)$ defines a rational map

$$
\theta_{r, m}: \mathcal{S} \rightarrow\left|\mathcal{O}_{X^{m}}(1, \ldots, 1)\right|
$$

sending the moduli point of $(E, S)$ to $\mathbb{D}_{E, S}$. Assume $X=\mathbb{G}$, where $\mathbb{G}$ is the Plücker embedding of the Grassmannian $G(r, r m)$. Then consider the pair $\left(\mathcal{U}^{*}, H\right)$, where $\mathcal{U}$ is the universal bundle of $\mathbb{G}$ and $H=H^{0}\left(\mathcal{U}^{*}\right)$. In this case the Plücker form of $\left(\mathcal{U}^{*}, H\right)$ is the zero locus

$$
\mathbb{D}_{\mathbb{G}} \in\left|\mathcal{O}_{\mathbb{G}^{m}}(1, \ldots, 1)\right|
$$

of a natural multilinear form related to $\mathbb{G}$. More precisely $\mathbb{G}$ is embedded in $\mathbb{P}\left(\wedge^{r} V\right)$, where $V=H^{*}$, and $\mathbb{D}_{\mathbb{G}}$ is the zero locus of the map

$$
d_{r, m}:\left(\wedge^{r} V\right)^{m} \rightarrow \wedge^{r m} V \cong \mathbf{C},
$$

induced by the wedge product. In the first part of the paper we prove that $\mathbb{G}$ is uniquely reconstructed from $\mathbb{D}_{\mathbb{G}}$ as soon as $m \geq 3$. We prove that:

Theorem Let $m \geq 3$ and let $x \in \mathbb{P}\left(\wedge^{r} V\right)$, then $x \in \mathbb{G}$ iff the following conditions hold true:

(1) $(x, \ldots, x) \in\left(\mathbb{P}\left(\wedge^{r} V\right)\right)^{m}$ is a point of multiplicity $m-1$ for $\mathbb{D}_{\mathbb{G}}$,

(2) $\operatorname{Sing}_{m-1}\left(\mathbb{D}_{\mathbb{G}}\right)$ has tangent space of maximal dimension at $(x, \ldots, x)$.

It follows essentially from this result that the previous map $\theta_{r, m}$ is generically injective, provided some suitable conditions are satisfied.

Indeed let $(E, S)$ be a pair as above and let $g_{E, S}: X \rightarrow \mathbb{G}_{E, S}$ be the classifying map in the Grassmannian $\mathbb{G}_{E, S}$ of $r$ dimensional subspaces of $S^{*}$. In section 4 we use the previous theorem to prove that:

Theorem $\theta_{r, m}$ is generically injective under the following assumptions:

(1) $\operatorname{Aut}(X)$ is trivial and $m \geq 3$,

(2) $g_{E, S}$ is a morphism birational onto its image,

(3) the determinant map $d_{E, S}: \wedge^{r} S \rightarrow H^{0}\left(\mathcal{O}_{X}(1)\right)$ is injective. 
However the main emphasis of this paper is on the case where $X \subset \mathbb{P}^{n}$ is a general curve of genus $g$ and $\mathcal{O}_{X}(1)$ has degree $r(m+g-1)$. Assuming this, we consider the moduli space $\mathcal{S}_{r}$ of pairs $\left(E, H^{0}(E)\right)$, where $E$ is a stable vector bundle of determinant $\mathcal{O}_{X}(1)$ and $h^{1}(E)=0$. Let $t$ be an $r$-root of $\mathcal{O}_{X}(1)$, then $\mathcal{S}_{r}$ is birational to $S U_{X}(r, 0)$ via the map

$$
\alpha: \mathcal{S}_{r} \rightarrow S U_{X}(r, 0)
$$

sending the moduli point of $\left(E, H^{0}(E)\right)$ to the moduli point of $E(-t)$. In the second half of the paper we prove that

$$
\theta_{r, m} \circ \alpha^{-1}=\beta \circ \theta_{r}
$$

where $\theta_{r}$ is the theta map of $S U_{X}(r, 0)$ and $\beta$ is a rational map. Moreover we prove that the assumptions of the latter theorem are satisfied if $X$ is general of genus $g>>r$. Then it follows that $\theta_{r}$ is generically injective as soon as $X$ is general of genus $g>>r$.

This completes the description of the proof of the main theorem of this paper. It seems interesting to use Plücker forms for further applications.

\section{PLÜCKER FORMS}

Let $V$ be a complex vector space of positive dimension $r m$ and let $\wedge^{r} V$ be the $r$-exterior power of $V$. On $\wedge^{r} V$ we consider the multilinear form

$$
d_{r, m}:\left(\wedge^{r} V\right)^{m} \rightarrow \wedge^{r m} V \simeq \mathbb{C},
$$

such that

$$
d_{r, m}\left(w_{1}, \ldots, w_{m}\right):=w_{1} \wedge \cdots \wedge w_{m} .
$$

Notice that $d_{r, m}$ is symmetric if $r$ is even and skew symmetric if $r$ is odd. We fix $m$ copies $V_{1}, \ldots, V_{m}$ of $V$ and the spaces $\mathbb{P}_{s}:=\mathbb{P}\left(\wedge^{r} V_{s}\right), s=1, \ldots, m$, of dimension $N:=\left(\begin{array}{c}r m \\ r\end{array}\right)-1$. Then we consider the Segre embedding

$$
\mathbb{P}_{1} \times \cdots \times \mathbb{P}_{m} \hookrightarrow \mathbb{P}^{(N+1) m-1}
$$

and its projections $\pi_{s}: \mathbb{P}_{1} \times \cdots \times \mathbb{P}_{m} \rightarrow \mathbb{P}_{s}, s=1, \ldots, m$. The form $d_{r, m}$ defines the following hyperplane section of $\mathbb{P}_{1} \times \cdots \times \mathbb{P}_{m}$ :

$$
\mathbb{D}_{r, m}:=\left\{\left(w_{1}, \ldots, w_{m}\right) \in \mathbb{P}_{1} \times \ldots \times \mathbb{P}_{m} \mid d_{r, m}\left(w_{1}, \ldots, w_{m}\right)=0\right\} .
$$

Definition 2.1. $\mathbb{D}_{r, m}$ is the Plücker form of $\mathbb{P}\left(\wedge^{r} V\right)^{m}$.

$\mathbb{D}_{r, m}$ is an element of the linear system $\left|\mathcal{O}_{\mathbb{P}_{1} \times \cdots \times \mathbb{P}_{m}}(1, \ldots, 1)\right|$, where

$$
\mathcal{O}_{\mathbb{P}_{1} \times \cdots \times \mathbb{P}_{m}}(1, \ldots, 1)=\pi_{1}^{*} O_{\mathbb{P}_{1}}(1) \otimes \ldots \otimes \pi_{m}^{*} O_{\mathbb{P}_{m}}(1) .
$$

Let $e_{1}, \ldots, e_{r m}$ be a basis of $V$ and let $\mathcal{I}$ be the set of all naturally ordered sets $I:=$ $i_{1}<\cdots<i_{r}$ of integers in $[1, r m]$. We fix in $\wedge^{r} V_{s}$ the basis

$$
e_{I}^{(s)}:=e_{i_{1}} \wedge \cdots \wedge e_{i_{r}}, \quad I=i_{1}<\cdots<i_{r} \in \mathcal{I}
$$

Then any vector of $\wedge^{r} V_{s}$ is of the form $\sum p_{I}^{(s)} e_{I}^{(s)}$, where the coefficients $p_{I}^{(s)}$ are the standard Plücker coordinates on $\mathbb{P}_{s}$. This implies that

$$
d_{r, m}\left(w_{1}, \ldots, w_{m}\right)=\sum_{I_{1} \cup \cdots \cup I_{m}=\{1, \ldots, r m\}} p_{I_{1}}^{(1)} \cdots p_{I_{m}}^{(m)} e_{I_{1}}^{(1)} \wedge \cdots \wedge e_{I_{m}}^{(m)}
$$

for each $\left(w_{1}, \ldots, w_{m}\right) \in\left(\wedge^{r} V\right)^{m}$. Note that, to give a decomposition

$$
I_{1} \cup \cdots \cup I_{m}=\{1, \ldots, r m\}
$$


as above, is equivalent to give a permutation $\sigma:\{1, \ldots, r m\} \rightarrow\{1, \ldots, r m\}$ which is strictly increasing on each of the intervals

$$
\mathbb{U}_{1}:=[1, r], \mathbb{U}_{2}:=[r+1,2 r], \ldots, \mathbb{U}_{m}:=[(m-1) r+1, m r] .
$$

Let $\mathcal{P}$ be the set of these permutations, then we conclude that

$$
d_{r, m}\left(w_{1}, \ldots, w_{m}\right)=\sum_{\sigma \in \mathcal{P}} \operatorname{sgn}(\sigma) p_{\sigma\left(\mathbb{U}_{1}\right)}^{(1)} \cdots p_{\sigma\left(\mathbb{U}_{M}\right)}^{(m)} e_{1} \wedge \cdots \wedge e_{r m}
$$

Assume that $w:=\left(w_{1}, \ldots, w_{m}\right) \in\left(\wedge^{r} V\right)^{m}$ is a vector defining the point $o \in \mathbb{P}_{1} \times \cdots \times \mathbb{P}_{m}$, we want to compute the Taylor series of $\mathbb{D}_{r, m}$ at $o$. Let $t:=\left(t_{1}, \ldots, t_{m}\right) \in\left(\wedge^{r} V\right)^{m}$, then we have the identity

$$
d_{r, m}\left(w_{1}+\epsilon t_{1}, \ldots, w_{m}+\epsilon t_{m}\right)=\sum_{k=0 \ldots m} \partial_{w}^{m-k} d_{r, m}(t) \epsilon^{k} .
$$

We will say that the function

$$
\partial_{w}^{m-k} d_{r, m}:\left(\wedge^{r} V\right)^{m} \rightarrow \mathbf{C},
$$

sending $t$ to the coefficient $\partial_{w}^{m-k} d_{r, m}(t)$ of $\epsilon^{k}$, is the $k$-th polar of $d_{r, m}$ at $w$, cf. [D]. Let $S:=s_{1}<\cdots<s_{k}$ be a strictly increasing sequence of $k$ elements of $M:=\{1, \ldots, m\}$. We will put $k:=|S|$. Moreover, for $w=\left(w_{1}, \ldots, w_{m}\right) \in\left(\wedge^{r} V\right)^{m}$, we define $w_{S}:=$ $w_{s_{1}} \wedge \cdots \wedge w_{s_{k}}$. Note that $\partial_{w}^{0}(t)=d\left(w_{1}, \ldots, w_{m}\right)$ for each $t$. If $m-k \geq 1$ it turns out that

$$
\partial_{w}^{m-k} d_{r, m}(t)=\sum_{|S|=k} \operatorname{sgn}\left(\sigma_{S}\right) w_{M-S} \wedge t_{S}
$$

where $\sigma_{S}: M \rightarrow M$ is the permutation $(1, \ldots, m) \rightarrow\left(j_{1}, \ldots, j_{m-k}, s_{1}, \ldots, s_{k}\right)$ such that $S=s_{1}<\cdots<s_{k}$ and $j_{1}<\cdots<j_{m-k}$.

Definition 2.2. Let $W:=\wedge^{r} V$ then

$$
q: \mathbb{P}\left(W^{m}\right) \rightarrow \mathbb{P}_{1} \times \ldots \times \mathbb{P}_{m}
$$

is the rational map sending the point defined by the vector $w=\left(w_{1}, \ldots, w_{m}\right)$ of $W^{m}$ to the $m$-tuple of points defined by the vectors $w_{1}, \ldots, w_{m}$.

Note that the pull-back of $d_{r, m}$ by $q$ is a homogeneous polynomial

$$
q^{*} d_{r, m} \in \operatorname{Sym}^{m} W^{*}=H^{0}\left(\mathcal{O}_{\mathbb{P}(W)}(m)\right) .
$$

We mention, without its non difficult proof, the following result

Proposition 2.3. $\partial_{w}^{m-k}\left(d_{r, m}\right)$ is the $k$-th polar form at $w$ of $q^{*} d_{r, m}$.

Let $\hat{o} \in \mathbb{P}\left(W^{m}\right)$ be the point defined by $w=\left(w_{1}, \ldots, w_{m}\right)$ and let $o=q(\hat{o})$. For the tangent spaces to $\mathbb{P}\left(W^{m}\right)$ at $\hat{o}$ and to $\mathbb{P}_{1} \times \cdots \times \mathbb{P}_{m}$ at $o$ one has

$$
\begin{array}{ll}
\circ & T_{\mathbb{P}\left(W^{m}\right), \hat{o}}=W^{m} /\langle w\rangle \\
\circ & T_{\mathbb{P}_{1} \times \cdots \times \mathbb{P}_{m}, o}=W /\left\langle w_{1}\right\rangle \oplus \cdots \oplus W /\left\langle w_{m}\right\rangle .
\end{array}
$$

Moreover the tangent map

$$
d q_{\hat{o}}: W^{m} /\langle w\rangle \longrightarrow W /\left\langle w_{1}\right\rangle \oplus \cdots \oplus W /\left\langle w_{m}\right\rangle
$$

is exactly the map sending

$$
\left(t_{1}, \ldots, t_{m}\right) \bmod \langle w\rangle \rightarrow\left(t_{1} \bmod \left\langle w_{1}\right\rangle, \ldots, t_{m} \bmod \left\langle w_{m}\right\rangle\right) .
$$


In particular we have

$$
\text { Ker } d q_{\hat{o}}=\left\{\left(c_{1} w_{1}, \ldots, c_{m} w_{m}\right),\left(c_{1}, \ldots, c_{m}\right) \in \mathbb{C}^{m}\right\} /\langle w\rangle .
$$

We can now use $d q_{\hat{o}}$ to study some properties of $\operatorname{Sing}\left(\mathbb{D}_{r, m}\right)$. We consider the $k$-osculating tangent cone $\mathcal{C}_{o}^{k} \subset T_{\mathbb{P}_{1} \times \cdots \times \mathbb{P}_{m}, o}$ to $\mathbb{D}_{r, m}$ at $o$.

Lemma 2.4. Keeping the above notations one has:

(1) $\operatorname{Sing}_{k}\left(\mathbb{D}_{r, m}\right)=\left\{o \in \mathbb{D}_{r, m} \mid \partial_{w}^{m-i}\left(d_{r, m}\right)=0, i \leq k-1\right\}$.

(2) $\mathcal{C}_{o}^{k}=d q_{\hat{o}}\left(\left\{t \in W^{m} \bmod \langle w\rangle \mid \partial_{w}^{m-i}\left(d_{r, m}\right)(t)=0, i \leq k\right\}\right)$

Proof. By the previous description of $d q_{\hat{o}}$ any one dimensional subspace $l$ of $T_{\mathbb{P}_{1} \times \cdots \times \mathbb{P}_{m}, o}$ is the isomorphic image by $d q_{\hat{o}}$ of the tangent space at $\hat{o}$ to an affine line

$$
L_{t}:=\{w+\epsilon t \mid \epsilon \in \mathbb{C}\} \subset \mathbb{P}\left(W^{m}\right),
$$

for some $t=\left(t_{1}, \ldots, t_{m}\right) \in W^{m}$. On the other hand the pull-back of the Taylor series of $\mathbb{D}_{r, m}$ to $L_{t}$ is

$$
d_{r, m}(w+\epsilon t)=\sum_{i=0 \ldots m} \partial_{w}^{m-i}\left(d_{r, m}\right)(t) \epsilon^{i}
$$

this implies (1) and (2).

Let $o \in \mathbb{P}_{1} \times \cdots \times \mathbb{P}_{m}$ be the point defined by the vector $\left(w_{1}, \ldots, w_{m}\right)$ and let $v \in$ $T_{\mathbb{P}_{1} \times \cdots \times \mathbb{P}_{m}, o}$ be a tangent vector to an arc of curve

$$
\left\{w_{1}+\epsilon t_{1}, \ldots, w_{m}+\epsilon t_{m}, \epsilon \in \mathbf{C}\right\} .
$$

Applying the lemma and the equality (3), it follows:

\section{Theorem 2.5.}

(i) $o \in \operatorname{Sing}_{k}\left(\mathbb{D}_{r, m}\right) \Longleftrightarrow w_{S}=0, \forall S \in \mathcal{I},|S|=m-k+1$.

(ii) $v$ is tangent to $\operatorname{Sing}_{k}\left(\mathbb{D}_{r, m}\right)$ at o iff

$$
\sum_{s \in S} \operatorname{sgn}\left(\sigma_{s}\right) w_{S-\{s\}} \wedge t_{s}=0, \forall S \in \mathcal{I},|S|=m-k+1,
$$

where $\sigma_{s}$ is the permutation of $S$ shifting $s$ to the bottom and keeping the natural order in $S-s$.

Proof. (i) By Lemma $2.4(1), o \in \operatorname{Sing}_{k}\left(\mathbb{D}_{r, m}\right)$ iff the $i$-th polar $\partial_{w}^{i}\left(d_{r, m}\right)$ is zero for $i \leq k-1$. This is equivalent to $w_{S}=0$ for $|S|=m-k+1$. (ii) As above, consider a tangent vector $v$ at $o$ to the arc of curve $\left\{w_{1}+\epsilon t_{1}, \ldots, w_{m}+\epsilon t_{m}, \epsilon \in \mathbf{C}\right\}$. By lemma 2.4 (2), $v$ is tangent to $\operatorname{Sing}_{k}\left(\mathbb{D}_{r, m}\right)$ at $o$ iff the coefficient of $\epsilon$ in $(w+\epsilon t)_{S}$ is zero, $\forall|S|=m-k+1$. This is equivalent to the condition $\sum_{s \in S} \operatorname{sgn}\left(\sigma_{s}\right) w_{S-\{s\}} \wedge t_{s}=0, \forall S \in \mathcal{I},|S|=m-k+1$.

Corollary 2.6. The Plücker form $\mathbb{D}_{r, m}$ has no point of multiplicity $\geq m$.

Proof. Assume $\mathbb{D}_{r, m}$ has multiplicity $\geq m$ at $o$. Then $w_{S}=0, \forall S$ with $|S|=1$. This means $w_{1}=\cdots=w_{m}=0$, which is impossible.

We are specially interested to the behaviour of $\mathbb{D}_{r, m}$ along its intersection with the diagonal

$$
\Delta \subset \mathbb{P}_{1} \times \cdots \times \mathbb{P}_{m} \subset \mathbb{P}^{(N+1) m-1}
$$


We recall that $\Delta$ spans the projectivized space of the symmetric tensors of $\left(\wedge^{r} V\right)^{\otimes m}$. Moreover, $\Delta$ is the $m$-Veronese embedding of $\mathbb{P}\left(\wedge^{r} V\right)$. If $r$ is odd $d_{r, m}$ is skew symmetric and $\mathbb{D}_{r, m}$ contains $\Delta$. If $r$ is even then

$$
\mathbb{D}_{r, m} \cdot \Delta
$$

is an interesting hypersurface of degree $m$ in the projective space $\Delta$. Applying Theorem 2.5 to a point $o$ in the diagonal, we have:

Corollary 2.7. Let $o \in \Delta$. Then:

(i) $o \in \operatorname{Sing}_{k}\left(\mathbb{D}_{r, m}\right) \Longleftrightarrow w^{\wedge m-k+1}=0$;

(ii) $v \in T_{\operatorname{Sing}_{k}\left(\mathbb{D}_{r, m}\right), o}$ if and only if

$$
\sum_{j \in S} \operatorname{sgn}\left(\sigma_{s}\right) w^{\wedge(m-k)} \wedge t_{j}=0, \quad \forall S \in \mathcal{I},|S|=m-k+1 .
$$

Remark 2.8. Let $o \in \Delta$ be as above, it follows from the corollary that:

$$
o \in \Delta \cap \operatorname{Sing}_{m-1}\left(\mathbb{D}_{r, m}\right) \Longleftrightarrow w \wedge w=0 .
$$

It is easy to see that $\Delta \subset \operatorname{Sing}_{m-1}\left(\mathbb{D}_{r, m}\right)$ if $r$ is odd. Let $r$ be even then

$$
\mathbb{G} \subset \Delta \cap \operatorname{Sing}_{m-1}\left(\mathbb{D}_{r, m}\right),
$$

where $\mathbb{G}$ is the Plücker embedding in $\Delta=\mathbb{P}\left(\wedge^{r} V\right)$ of the Grassmannian $G(r, V)$. However it is not true that the equality holds in the latter case. In fact the equation $w \wedge w=0$ defines $\mathbb{G}$ if and only if $r=2$, see [Ha1].

\section{PLÜCKER FORMS AND GRASSMANNIANS}

In this section we will keep the notation $\mathbb{G}$ for the Plücker embedding of $G(r, V)$. Our purpose is now to show that $\mathbb{G}$ is uniquely reconstructed from $\mathbb{D}_{r, m}$ and the diagonal $\Delta$. More precisely we will show the following:

Theorem 3.1. Let $m \geq 3$, then

$$
\mathbb{G}=\left\{o \in \Delta \cap \operatorname{Sing}_{m-1}\left(\mathbb{D}_{r, m}\right) \mid \operatorname{dim} T_{\text {Sing }_{m-1}\left(\mathbb{D}_{r, m}\right), o} \text { is maximal. }\right\}
$$

For the proof we need some preparation. The following result of linear algebra will be useful: let $E$ be a vector space of dimension $d$ and let $w \in \wedge^{r} E$ be a non zero vector. Consider the linear map

$$
\mu_{w}^{s}: \wedge^{s} E \rightarrow \wedge^{r+s} E
$$

sending $t$ to $w \wedge t$. We have:

Proposition 3.2. Let $d-2 r \geq s$, then $\mu_{w}^{s}$ has rank $\geq\left(\begin{array}{c}d-r \\ s\end{array}\right)$ and the equality holds if and only if the vector $w$ is decomposable.

Proof. We fix, with the previous notations, a basis $\left\{e_{1}, \ldots, e_{d}\right\}$ of $E$ and the corresponding basis $\left\{e_{I}, I=i_{1}<\cdots<i_{r}\right\}$ of $\wedge^{r} E$. Let $e_{I_{0}}:=e_{1} \wedge \cdots \wedge e_{r}$ so that $I_{0}=1<2<\cdots<r$. Since $w$ is non zero we can assume that $w=e_{I_{0}}+\sum_{I \neq I_{0}} a_{I} e_{I}$. Let $W^{-}, W^{+}$be the subspaces of $E$ respectively generated by $\left\{e_{1}, \ldots, e_{r}\right\}$ and $\left\{e_{r+1}, \ldots, e_{d}\right\}$. Then we have the direct sum decomposition

$$
\wedge^{r+s} E=E^{+} \oplus E^{-}
$$


where $E^{+}$and $E^{-}$are defined as follows:

$$
E^{+}=\left\{e_{I_{0}} \wedge u, u \in \wedge^{s} W^{+}\right\} \text {and } E^{-}=\left\{\sum_{i=1, \ldots, r} e_{i} \wedge v_{i}, v_{i} \in \wedge^{r+s-1} E\right\} .
$$

Let $p^{+}: \wedge^{r+s} E \rightarrow E^{+}$be the projection map. Since $w=e_{I_{0}}+\sum_{I \neq I_{0}} a_{I} e_{I}$, the map

$$
\left(p^{+} \circ \mu_{w}^{s}\right)_{\left.\right|_{\wedge^{s} W^{+}}}: \wedge^{s} W^{+} \rightarrow E^{+}
$$

is just the map $u \rightarrow e_{I_{0}} \wedge u$, in particular it is an isomorphism. This implies that

$$
\operatorname{rank} \mu_{w}^{s} \geq \operatorname{rank}\left(p_{r} \circ \mu_{w}^{s}\right)=\operatorname{dim} \wedge^{s} W^{+}=\left(\begin{array}{c}
d-s \\
r
\end{array}\right) .
$$

Let $w$ be decomposable, then there is no restriction to assume $w=e_{I_{o}}$ and it follows $\operatorname{dim} \operatorname{Im} \mu_{w}^{s}=\left(\begin{array}{c}d-r \\ s\end{array}\right)$. Now let's assume that $w$ is not decomposable. To complete the proof it suffices to show that, in this case,

$$
\operatorname{dim} \operatorname{Im} \mu_{w}^{s}>\left(\begin{array}{c}
d-s \\
r
\end{array}\right)
$$

By the above remarks $\mu_{w}^{s}$ is injective on $\wedge^{s} W^{+}$. Hence the inequality (5) holds iff

$$
\mu_{w}^{s}\left(\wedge^{s} W^{+}\right) \neq \operatorname{Im} \mu_{w}^{s} .
$$

On the other hand $p^{+} \circ \mu_{w}^{s}: \wedge^{r} W^{+} \rightarrow E^{+}$is an isomorphism and $\operatorname{dim} \wedge^{s} W^{+}=\left(\begin{array}{c}d-r \\ s\end{array}\right)$. Therefore inequality (6) is satisfied iff there exists a vector $\tau \in \wedge^{r+s} E$ such that

$$
0 \neq \tau \in \operatorname{Im} \mu_{w}^{s} \cap \operatorname{Ker} p^{+} .
$$

So, to complete the proof, it remains to show the following:

Claim Let $d-2 r \geq s$ and $w$ be not decomposable. Then there exists a vector $\tau$ as above.

Proof By induction on $s$. If $s=1$ we have $\operatorname{dim} \operatorname{Im} \mu_{w}^{1} \geq d-r$. It is proved in [G] Prop. 6.27, that the strict inequality holds iff $w$ is not decomposable. Hence we have $\operatorname{dim} \operatorname{Im} \mu_{w}^{1}>d-r$ and there exists a non zero $\tau \in \operatorname{Im} \mu_{w}^{1} \cap \operatorname{Ker} p^{+}$.

Now assume that $\tau \in \operatorname{Im} \mu_{w}^{s-1}$ is a non zero vector satisfying the induction hypothesis. Let $N=\{v \in E \mid \tau \wedge v=0\}$. Then $N$ is the Kernel of the map $\mu_{\tau}^{1}: E \rightarrow \wedge^{r+s-1} E$ and, by the first part of the proof, $\operatorname{dim} N \leq r+s-1$. Since we are assuming $s+r \leq d-r$, it follows that we can find a vector $e_{k} \in\left\{e_{1}, \ldots, e_{d}\right\}$ such that

$$
e_{k} \wedge e_{1} \wedge \cdots \wedge e_{r} \neq 0 \text { and } e_{k} \notin N \text {. }
$$

Then for such a vector we have

$$
0 \neq e_{k} \wedge \tau=\sum b_{J} e_{k} \wedge e_{J}, \quad|J|=r+s-1, \quad I_{0} \not \subset\{J \cup k\}
$$

and, moreover, $e_{k} \wedge \tau \in \operatorname{Im} \mu_{w}^{s}$. Hence the claim follows.

From now on we will assume $m \geq 3$. Moreover we identify $\wedge^{r} V$ to its image via the diagonal embedding

$$
\delta: \wedge^{r} V \rightarrow\left(\wedge^{r} V\right)^{m}
$$

sending $w$ to $\delta(w):=(w, \ldots, w)$. Let $o \in \Delta$ be the point defined by $w=(w, \ldots, w)$. From Corollary 2.7 (i), we have that

$$
\Delta \cap \operatorname{Sing}_{m-1}\left(\mathbb{D}_{r, m}\right)=\{o \in \Delta \mid w \wedge w=0\} .
$$


Moreover let $\left(t_{1}, \ldots, t_{m}\right) \in\left(\wedge^{r} V\right)^{m}$, and let $v$ be a tangent vector at $o$ to

$$
\left\{\left(w+\epsilon t_{1}, \ldots, w+\epsilon t_{m}\right), \epsilon \in \mathbf{C}\right\} \subset \mathbb{P}_{1} \times \cdots \times \mathbb{P}_{m},
$$

it follows from Corollary 2.7 that $v$ is tangent to $\operatorname{Sing}_{m-1}\left(\mathbb{D}_{r, m}\right)$ at $o$ iff

$$
w \wedge t_{j}+t_{i} \wedge w=0, \quad 1 \leq i<j \leq m,
$$

in the vector space $\wedge^{2 r} V$. Let

$$
\vartheta:\left(\wedge^{r} V\right)^{m} \rightarrow\left(\wedge^{r} V /\langle w\rangle\right)^{m}
$$

be the natural quotient map, where $\left(\wedge^{r} V /\langle w\rangle\right)^{m}=T_{\mathbb{P}_{1} \times \cdots \times \mathbb{P}_{m}, o}$. Consider

$$
T_{o}:=\left\{\left(t_{1}, \ldots, t_{m}\right) \in\left(\wedge^{r} V\right)^{m} \mid w \wedge t_{j}+t_{i} \wedge w=0,1 \leq i<j \leq m\right\}
$$

and note that, by the latter remark, one has

$$
\vartheta^{-1}\left(T_{\text {Sing }_{m-1}\left(\mathbb{D}_{r, m}\right), o}\right)=T_{o} .
$$

For any point $o \in \Delta \cap \operatorname{Sing}_{m-1}\left(\mathbb{D}_{r, m}\right)$ we define

$$
c_{o}=\text { codimension of } T_{\operatorname{Sing}_{m-1}\left(\mathbb{D}_{r, m}\right), o} \text { in } T_{\mathbb{P}_{1} \times \cdots \times \mathbb{P}_{m}, o},
$$

Since $\vartheta$ is surjective, it is clear that $c_{o}$ is the codimension of $T_{o}$ in $\left(\wedge^{r} V\right)^{m}$.

Lemma 3.3. Let $c_{o}$ be as above and let $B:=\left(\begin{array}{c}(m-1) r \\ r\end{array}\right)$, then

(i) $c_{o} \geq m B$ if $r$ is even and $m \geq 3$,

(ii) $c_{o} \geq(m-1) B$ if $r$ is odd and $m \geq 3$,

(iii) $c_{o}=m-1$ if $m \leq 2$.

Moreover the equality holds in (i) and (ii) iff $w$ is a decomposable vector.

Proof. Let $w^{\perp} \subset \wedge^{r} V$ be the orthogonal space of $w=(w, \ldots, w)$ with respect to the bilinear form

$$
\wedge: \wedge^{r} V \times \wedge^{r} V \rightarrow \wedge^{2 r} V
$$

Moreover let $N \subset\left(\wedge^{r} V\right)^{m}$ be the subspace defined by the equations

$$
(-1)^{r} t_{i}+t_{j}=0,1 \leq i<j \leq m .
$$

It is easy to check that

$$
T_{o}=N+\left(w^{\perp}\right)^{m} .
$$

Let $m \geq 3$ then $N$ is the diagonal subspace if $r$ is odd and $N=(0)$ if $r$ is even. By Proposition 3.2, we have that codim $w^{\perp} \geq B$ and moreover the equality holds iff $w$ is a decomposable vector. This implies (i), (ii) and the latter statement. Let $m \leq 2$ then $N$ is either the diagonal subspace or the space of pairs $(t,-t), t \in \wedge^{r} V$. Arguing as above it follows that $c_{o}=(m-1) B$, i.e. $c_{o}=m-1$. This completes the proof.

Proof of Theorem 3.1

The proof is now immediate: let $o \in \Delta \cap \operatorname{Sing}_{m-1}\left(\mathbb{D}_{r, m}\right)$. It is obvious that the codimension $c_{o}$ is minimal iff $\operatorname{dim} T_{\mathrm{Sing}_{m-1}\left(\mathbb{D}_{r, m}\right), o}$ is maximal. Assume $m \geq 3$, by Lemma $3.3 c_{o}$ is minimal iff $o \in \mathbb{G}$.

Keeping our usual notations we have

$$
\mathbb{G}^{m} \subset \mathbb{P}_{1} \times \cdots \times \mathbb{P}_{m} \subset \mathbb{P}^{(N+1)^{m}-1},
$$


where the latter inclusion is the Segre embedding and $\mathbb{G}$ is the previous Plücker embedding. The restriction of $\mathbb{D}_{r, m}$ to $\mathbb{G}^{m}$ has a geometric interpretation given in the next lemma.

Let $o=\left(w_{1}, \ldots, w_{m}\right) \in \mathbb{G}^{m}$. Then we have $w_{s}:=v_{1}^{(s)} \wedge \cdots \wedge v_{r}^{(s)}$, where $v_{1}^{(s)}, \ldots, v_{r}^{(s)} \in V_{s}$ and $s=1, \ldots, m$. In particular $w_{s}$ is a decomposable vector, so it defines a point $l_{s}$ in $\mathbb{G}$. The vector space corresponding to $l_{s}$ is generated by the basis $v_{1}^{s}, \ldots, v_{r}^{s}$. We will denote its projectivization by $L_{s}$.

Lemma 3.4. The following conditions are equivalent:

(i) $o \in \mathbb{D}_{r, m}$,

(ii) $w_{1} \wedge \cdots \wedge w_{m}=0$,

(iii) $\left\{v_{i}^{j}\right\}, 1 \leq i \leq r, 1 \leq j \leq m$, is not a basis of $V$,

(iv) there exists a hyperplane in $\mathbb{P}(V)$ containing $L_{1} \cup \cdots \cup L_{m}$.

Proof. Immediate.

Lemma 3.5. $\mathbb{D}_{r, m}$ cuts on $\mathbb{G}^{m}$ an integral hyperplane section.

Proof. Consider the correspondence

$$
I=\left\{\left(l_{1}, \ldots, l_{m}, H\right) \in \mathbb{G}^{m} \times \mathbb{P}\left(V^{*}\right) \mid L_{1} \cup \cdots \cup L_{m} \subset H\right\},
$$

and its projections $p_{1}: I \rightarrow \mathbb{G}^{m}$ and $p_{2}: I \rightarrow \mathbb{P}\left(V^{*}\right)$. Note that the fibre of $p_{2}$ at any $H$ is the product of Grassmannians of $r-1$ spaces in $H$, which is irreducible. Hence $I$ is irreducible. On the other hand we have $p_{1}(I)=\mathbb{D}_{r, m} \cap \mathbb{G}^{m}$ by Lemma 3.4 (iv). Hence the latter intersection is irreducible. Since $O_{\mathbb{G}^{m}}(1)$ is not divisible in $\operatorname{Pic}\left(\mathbb{G}^{m}\right)$, it follows that $\mathbb{D}_{r, m} \cdot \mathbb{G}^{m}$ is integral.

On $\mathbb{G}$ we consider the universal bundle $\mathcal{U}_{r}$. We recall that $\mathcal{U}_{r}$ is uniquely defined by its Chern classes, unless $m=2$. Let $l \in \mathbb{G}$ and let $L \subset \mathbb{P}(V)$ be the space corresponding to $l$. Then the fibre of $\mathcal{U}_{r}^{*}$ at $l$ is $H^{0}\left(\mathcal{O}_{L}(1)\right)$, moreover $H^{0}\left(\mathcal{U}_{r}{ }^{*}\right)=V^{*}=H^{0}\left(O_{\mathbb{P}(V)}(1)\right)$. Let $\pi_{s}: \mathbb{G}^{m} \rightarrow \mathbb{G}$ be the projection onto the $s$-th factor. On $\mathbb{G}^{m}$ we consider the vector bundle of rank $\mathrm{rm}$

$$
\mathcal{F}:=\bigoplus_{s=1, \ldots, m} \pi_{s}^{*} \mathcal{U}_{r}^{*}
$$

For any point $o=\left(l_{1}, \ldots, l_{m}\right) \in \mathbb{G}^{m}$, we have

$$
\mathcal{F}_{o}=\left(\mathcal{U}_{r}^{*}\right)_{l_{1}} \oplus . . \oplus\left(\mathcal{U}_{r}^{*}\right)_{l_{m}}=H^{0}\left(O_{L_{1}}(1)\right) \oplus \ldots \oplus H^{0}\left(O_{L_{m}}(1)\right) .
$$

In particular the natural evaluation map

$$
e v^{m}: V^{*} \otimes \mathcal{O}_{\mathbb{G}^{m}} \rightarrow \mathcal{F}
$$

is a morphism of vector bundles of the same rank $\mathrm{rm}$.

Definition 3.6. $\mathbb{D}_{\mathbb{G}}$ is the degeneracy locus of $e v^{m}$.

Theorem 3.7. $\mathbb{D}_{\mathbb{G}}=\mathbb{D}_{r, m} \cdot \mathbb{G}^{m}$.

Proof. Let $o=\left(l_{1}, \ldots, l_{m}\right) \in \mathbb{G}^{m}$, then $e v_{o}^{m}$ is the natural restriction map

$$
H^{0}\left(O_{\mathbb{P}(V)}(1)\right) \rightarrow H^{0}\left(O_{L_{1}}(1)\right) \oplus \ldots \oplus H^{0}\left(O_{L_{m}}(1)\right) .
$$

Note that $e v_{o}^{m}$ is an isomorphism iff $L_{1} \cup \cdots \cup L_{m}$ is not in a hyperplane of $\mathbb{P}(V)$. This implies that $\mathbb{D}_{\mathbb{G}}$ is a divisor. Moreover $\mathbb{D}_{\mathbb{G}}=\mathbb{D}_{r, m} \cap \mathbb{G}^{m}$ by Lemma 3.4 and $\mathbb{D}_{\mathbb{G}} \in$ $\left|O_{\mathbb{G}^{m}}(1, . ., 1)\right|$. Hence $\mathbb{D}_{\mathbb{G}}=\mathbb{D}_{r, m} \cdot \mathbb{G}^{m}$. 


\section{PlÜCKER FORMS AND MODULI OF VECTOR BUNDLES}

In this section we consider any integral, smooth projective variety $X \subset \mathbb{P}^{n}$ of dimension $d \geq 1$. We assume that $X$ is linearly normal and not degenerate.

Definition 4.1. $(E, S)$ is a good pair on $X$ if

(i) $E$ is a vector bundle of rank $r$ on $X$,

(ii) $\operatorname{det} E \cong \mathcal{O}_{X}(1)$,

(iii) $S \subset H^{0}(E)$ is a subspace of dimension $r m$,

(iv) $E$ is globally generated by $S$,

(v) the classifying map of $(E, S)$ is a morphism birational onto its image.

Given $(E, S)$ we have the dual space $V:=S^{*}$ and its Plücker form

$$
\mathbb{D}_{r, m} \subset \mathbb{P}\left(\wedge^{r} V\right)^{m} \text {. }
$$

We want to use it. Let us fix preliminarily some further notations:

\section{Definition 4.2.}

(i) $\mathbb{G}_{E, S}$ is the Plücker embedding of the Grassmannian $G(r, V)$,

(ii) $\mathcal{U}_{E, S}$ is the universal bundle of $\mathbb{G}_{E, S}$,

(iii) $d_{E, S}: \wedge^{r} S \rightarrow H^{0}\left(\mathcal{O}_{X}(1)\right)$ is the standard determinant map,

(iv) $\lambda_{E, S}: \mathbb{P}^{n} \rightarrow \mathbb{P}\left(\wedge^{r} V\right)$ is the projectivized dual of $d_{E, S}$,

(v) $g_{E, S}: X \rightarrow \mathbb{G}_{E, S}$ is the classifying map defined by $S$.

We recall that $g_{E, S}$ associates to $x \in X$ the parameter point of the space $\operatorname{Im} e v_{x}^{*}$, where $e v: S \otimes \mathcal{O}_{X} \rightarrow E$ is the evaluation map. It is well known that $g_{E, S}$ is defined by the subspace $\operatorname{Im} d_{E, S}$ of $H^{0}\left(\mathcal{O}_{X}(1)\right)$, in particular

$$
g_{E, S}=\lambda_{E, S \mid x} \text {. }
$$

Since $E$ is globally generated by $S$ and $g_{E, S}$ is a birational morphism, the next three lemmas describe standard properties.

Lemma 4.3. One has $E \cong \lambda_{E, S}^{*} \mathcal{U}_{E, S}^{*}$ and $S=\lambda_{E, S}^{*} H^{0}\left(\mathcal{U}_{E, S}^{*}\right)$ for any good pair $(E, S)$.

We say that the good pairs $\left(E_{1}, S_{1}\right),\left(E_{2}, S_{2}\right)$ are isomorphic if there exists an isomorphism $u: E_{1} \rightarrow E_{2}$ such that $u^{*} S_{1}=S_{2}$.

Lemma 4.4. Let $\left(E_{1}, S_{1}\right)$ and $\left(E_{2}, S_{2}\right)$ be good pairs. Then the following conditions are equivalent:

(i) $d_{E_{1}, S_{1}}=d_{E_{2}, S_{2}} \circ\left(\wedge^{r} \alpha\right)$ for some isomorphism $\alpha: S_{1} \rightarrow S_{2}$.

(ii) $f^{*} E_{1} \cong E_{2}$ and $f^{*} S_{1}=S_{2}$ for some automorphism $f \in \operatorname{Aut}(X)$.

Proof. (i) $\Rightarrow$ (ii) The projectivized dual of $\wedge^{r} \alpha$ induces an isomorphism $a: \mathbb{G}_{E_{2}, S_{2}} \rightarrow$ $\mathbb{G}_{E_{1}, S_{1}}$ such that $g_{E_{1}, S_{1}}=a \circ g_{E_{2}, S_{2}}$. On the other hand, $g_{E_{i}, S_{i}}: X \rightarrow \mathbb{G}_{E_{i}, S_{i}}$ is a morphism birational onto its image for $i=1,2$. Hence $a$ lifts to an automorphism $f: X \rightarrow X$ with the required properties. (ii) $\Rightarrow$ (i) It suffices to put $\alpha=f^{*}$.

Let $\rho_{i}: X^{m} \rightarrow X$ be the projection onto the $i$-th factor of $X^{m}$. Then

$$
e v_{E, S}: S \otimes O_{X^{m}} \rightarrow \bigoplus_{i=1, \ldots, m} \rho_{i}^{*} E:=\mathcal{E}
$$


is the morphism defined as follows. Let $U \subset X^{m}$ be open, we observe that $\mathcal{E}(U)=$ $E(U)^{m}$. Then we define the map $e v_{E, S}(U): S \rightarrow E(U)^{m}$ as the natural restriction map. Since $e v_{E, S}$ is a morphism of vector bundles of the same rank, its degeneracy locus is either $X^{m}$ or a divisor

$$
\mathbb{D}_{E, S} \in\left|O_{X^{m}}(1, . ., 1)\right| \text {. }
$$

Definition 4.5. We will say that the divisor $\mathbb{D}_{E, S}$ is the determinant divisor, or the Plücker form, of the pair $(E, S)$.

If the previous locus is $X^{m}$ we will say that $(E, S)$ has no Plücker form.

Lemma 4.6. Let $\left(E_{1}, S_{1}\right)$ and $\left(E_{2}, S_{2}\right)$ be isomorphic good pairs. Then $\mathbb{D}_{E_{2}, S_{2}}=\mathbb{D}_{E_{1}, S_{1}}$.

Proof. Let $u: E_{1} \rightarrow E_{2}$ be an isomorphism such that $u^{*} S_{2}=S_{1}$. Then, by taking the pull back of $u$ to $e v_{E_{1}, S_{1}}: S_{1} \otimes \mathcal{O}_{X} \rightarrow \mathcal{E}_{1}$, we obtain $e v_{E_{2}, S_{2}}$. This implies that $\mathbb{D}_{E_{1}, S_{1}}=\mathbb{D}_{E_{2}, S_{2}}$.

Remark 4.7. Note that $\mathbb{D}_{E, S}$ contains the multidiagonal $\Delta_{m}$, i.e. the set of all the points $\left(x_{1}, \ldots, x_{m}\right) \in X^{m}$ such that $x_{i}=x_{j}$ for some distinct $i, j \in\{1, \ldots, m\}$. Moreover, $\Delta_{m}$ is a divisor in $X^{m}$ iff $\operatorname{dim} X=1$. In this case $\mathbb{D}_{E, S}$ is reducible:

Proposition 4.8. Assume that $X$ is a curve, then

$$
\mathbb{D}_{E, S}=(r+\epsilon) \Delta_{m}+\mathbb{D}_{E, S}^{*} \text {. }
$$

where $\epsilon \geq 0$ and the support of the divisor $\mathbb{D}_{E, S}^{*}$ is the Zariski closure of the set

$$
\left\{\left(x_{1}, . ., x_{m}\right) \in X^{m}-\Delta_{m} \mid \exists s \in S \quad s\left(x_{i}\right)=0, \quad i=1, . ., m .\right\}
$$

Proof. Let $x=\left(x_{1}, \ldots, x_{m}\right) \in \Delta_{m}$. Then $e v_{E, S}$ has rank $\leq r m-r$ at $x$. This implies that $x$ is a point of multiplicity $\geq r$ of the determinant divisor $\mathbb{D}_{E, S}$. Hence $\Delta_{m}$ is a component of $\mathbb{D}_{E, S}$ of multiplicity $\geq r$. This implies the statement.

Actually, $\epsilon=0$ if $E$ is a general semistable vector bundle on the curve $X$. It is enough to verify this property in the case $E=L^{\oplus r}$ and $S=H^{0}(E)$, where $L$ is a general line bundle on $X$ of degree $m+g-1$. In this case the Plücker form of $(E, S)$ is indeed $r$ times the Plücker form of $\left(L, H^{0}(L)\right)$.

It is also non difficult to compute that $\mathbb{D}_{E, S}-r \Delta_{m}$ is numerically equivalent to $a^{*} r \Theta$, where $a: X^{m} \rightarrow \operatorname{Pic}^{m}(X)$ is the natural Abel map and $\Theta \subset \operatorname{Pic}^{m}(X)$ is a theta divisor. Finally we consider the commutative diagram

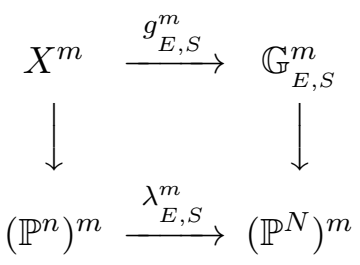

where the vertical arrows are the inclusion maps.

Lemma 4.9. Let $\mathbb{D}_{E, S}$ be the Plücker form of a good pair $(E, S)$, then

$$
\mathbb{D}_{E, S}=\left(\lambda_{E, S}^{m}\right)^{*} \mathbb{D}_{r, m}
$$

Proof. Lifting by $g_{E, S}^{m}$ the map $e v^{m}: V \otimes \mathcal{O}_{\mathbb{G}_{E, S}} \rightarrow \bigoplus_{i=1, \ldots, m} \pi_{s}^{*} \mathcal{U}_{E, S^{\prime}}$, one obtains the map $e v_{E, S}: S \otimes O_{X^{m}} \rightarrow \bigoplus_{i=1, \ldots, m} \rho_{i}^{*} E$. From the commutativity of the above diagram it follows that $\mathbb{D}_{E, S}=\left(\lambda_{E, S}^{m}\right)^{*} \mathbb{D}_{r, m}=\left(g_{E, S}^{m}\right)^{*} \mathbb{D}_{\mathbb{G}_{E, S}}$. 
To a good pair $(E, S)$ we have associated its Plücker form $\mathbb{D}_{E, S}$. Now we want to prove that, under suitable assumptions, a good pair $(E, S)$ is uniquely reconstructed from $\mathbb{D}_{E, S}$. To this purpose we define the following projective variety in the ambient space $\mathbb{P}^{n}$ of $X$.

Definition 4.10. $\Gamma_{E, S}$ is the closure of the set of points $x \in \mathbb{P}^{n}$ such that:

(i) $\mathbb{D}_{E, S}$ has multiplicity $m-1$ at the point $o=(x, \ldots, x) \in\left(\mathbb{P}^{n}\right)^{m}$,

(ii) the tangent space to $\operatorname{Sing}\left(\mathbb{D}_{E, S}\right)$ at o has maximal dimension.

Theorem 4.11. Assume that $d_{E, S}$ is injective and $m \geq 3$. Then:

(i) $\Gamma_{E, S}$ is a cone in $\mathbb{P}^{n}$ with directrix the Grassmannian $\mathbb{G}_{E, S}$,

(ii) the vertex of the cone $\Gamma_{E, S}$ is the center of the projection $\lambda_{E, S}$.

Proof. Since $\lambda_{E, S}$ is the projective dual of $d_{E, S}$, the tensor product map

$$
d_{E, S}^{\otimes m}:\left(\wedge^{r} S\right)^{\otimes m} \rightarrow H^{0}\left(\mathcal{O}_{X}(1)\right)^{\otimes m}
$$

is precisely the pull-back map

$$
\left(\lambda_{E, S}^{m}\right)^{*}: H^{0}\left(\mathcal{O}_{\left(\mathbb{P}\left(\wedge^{r} V\right)\right)^{m}}(1, \ldots, 1)\right) \rightarrow H^{0}\left(\mathcal{O}_{\left(\mathbb{P}^{n}\right)^{m}}(1, \ldots, 1)\right) .
$$

Moreover it is injective. Let $F \in H^{0}\left(\mathcal{O}_{\left(\mathbb{P}\left(\wedge^{r} V\right)\right)^{m}}(1, \ldots, 1)\right)$ be the polynomial of multidegree $(1, \ldots, 1)$ defining $\mathbb{D}_{r, m}$. Then we can choose coordinates on $\left(\mathbb{P}\left(\wedge^{r} V\right)\right)^{m}$ and $\left(\mathbb{P}^{n}\right)^{m}$ so that $d_{E, S}^{\otimes m}(F)=F$. Assume that $\lambda_{E, S}^{m}$ is a morphism at the point $o \in\left(\mathbb{P}^{n}\right)^{m}$, then it follows that:

(a) $\lambda_{E, S}^{m}(o) \in \operatorname{Sing}_{m-1}\left(\mathbb{D}_{r, m}\right)$ iff $o \in \operatorname{Sing}_{m-1}\left(\mathbb{D}_{E, S}\right)$,

(b) the codimension is equal for the tangent spaces to $\operatorname{Sing}_{m-1}\left(\mathbb{D}_{r, m}\right)$ at $\lambda_{E, S}^{m}(o)$ and to $\operatorname{Sing}_{m-1}\left(\mathbb{D}_{E, S}\right)$

at $o$.

Assume that $o=(x, \ldots, x)$ is a diagonal point in $\left(\mathbb{P}^{n}\right)^{m}$. Then $x \in \Gamma_{E, S}$ iff $o$ satisfies (i) and (ii) in Definition 4.10. By (a) and (b), conditions (i) and (ii) hold true for $o$ iff they hold true for $\lambda_{E, S}^{m}(o)$ as a point of $\mathbb{D}_{r, m}$. Finally, by Theorem 3.1, $\lambda_{E, S}(o)$ satisfies (i) and (ii) iff $x$ belongs to the Grassmannian $\mathbb{G}_{E, S}$. Hence $\Gamma_{E, S}$ is a cone over $\mathbb{G}_{E, S}$ with vertex the center of $\lambda_{E, S}$.

We are now able to show the main result of the current section.

Theorem 4.12. Let $\left(E_{1}, S_{1}\right)$ and $\left(E_{2}, S_{2}\right)$ be good pairs defining the same Plücker form $\mathbb{D} \subset$ $\left(\mathbb{P}^{n}\right)^{m}$. Assume that $m \geq 3$ and $d_{E_{i}, S_{i}}$ is injective for any $i=1,2$, then there exists $f \in$ $\operatorname{Aut}(X)$ such that $f^{*} E_{2} \cong E_{1}$ and $f^{*} S_{2}=S_{1}$.

Proof. Let $\Gamma$ be the closure of the set of diagonal points $o=(x, \ldots, x) \in \mathbb{D}$ of multiplicity $m-1$ and tangent space $T_{\text {Sing }_{m-1}}(\mathbb{D}), o$ of maximal dimension. By Theorem $4.11, \Gamma$ is a cone in $\mathbb{P}^{n}$ : its directrix is the Grassmannian $G_{E_{i}, S_{i}}$ and its vertex is the center of the projection $\lambda_{E_{i}, S_{i}}$, both for $i=1$ and $i=2$. Since the projection maps $\lambda_{E_{i}, S_{i}}$ have the same center, there exist an isomorphism $\sigma: G_{E_{2}, S_{2}} \rightarrow G_{E_{1}, S_{1}}$ such that $\lambda_{E_{1}, S_{1}}=\sigma \circ \lambda_{E_{2}, S_{2}}$. Since $m \geq 3$, then $\sigma=\wedge^{r} \alpha^{*}$ for an isomorphism $\alpha: S_{1} \rightarrow S_{2}$, see [Ha1] p.122. Then, applying Lemma 4.4, it follows $f^{*} E_{1} \cong E_{2}$ and $f^{*} S_{1}=S_{2}$ for some $f \in \operatorname{Aut}(X)$.

To conclude this section we briefly summarize, in a general statement, how to deduce from the previous results the generic injectivity of some natural maps, defined on a moduli space of good pairs as above. Therefore we assume that a coarse moduli space 
$\mathcal{S}$ exists for the family of good pairs $(E, S)$ under consideration. This is, for instance the case when $E$ is stable with respect to the polarization $\mathcal{O}_{X}(1)$ and $S=H^{0}(E)$. Then there exists a natural map

$$
\theta_{r, m}: \mathcal{S} \rightarrow\left|\mathcal{O}_{X^{m}}(1, \ldots, 1)\right|
$$

sending the moduli point of $(E, S)$ to its determinant divisor $\mathbb{D}_{E, S}$. Let $\left(E_{1}, S_{1}\right)$ and $\left(E_{2}, S_{2}\right)$ be good pairs as above defining two general points of $\mathcal{S}$. Assume that $\mathbb{D}_{E_{1}, S_{1}}=$ $\mathbb{D}_{E_{2}, S_{2}}$. Then we know from Theorem 4.12 that then $\left(E_{1}, S_{1}\right)$ and $\left(E_{2}, S_{2}\right)$ are isomorphic if $m \geq 3, \operatorname{Aut}(X)=1$ and

$$
d_{E_{i}, S_{i}}: \wedge^{r} S_{i} \rightarrow H^{0}\left(\mathcal{O}_{X}(1)\right) .
$$

is injective. This implies the next statement:

Theorem 4.13. Let $m \geq 3$ and $\operatorname{Aut}(X)=1$. Assume $d_{E, S}: \wedge^{r} S \rightarrow H^{0}\left(\mathcal{O}_{X}(1)\right)$ is injective for good pairs $(E, S)$ with moduli in a dense open subset of $\mathcal{S}$. Then $\theta_{r, m}$ is generically injective.

\section{PLÜCKER FORMS AND THE THETA MAP OF $S U_{X}(r, 0)$}

Now we apply the preceding arguments to study the theta map of the moduli space $S U_{X}(r, 0)$ of semistable vector bundles of rank $r$ and trivial determinant over a curve $X$ of genus $g \geq 2$. By definition the theta map

$$
\theta_{r}: S U_{X}(r, 0) \rightarrow \mathbb{P}\left(H^{0}(\mathcal{L})^{*}\right)
$$

is just the rational map defined by the ample generator $\mathcal{L}$ of $S U_{X}(r, 0)$. We prove our main result:

Theorem 5.1. Let $X$ be general and $g>>r$, then $\theta_{r}$ is generically injective.

To prove the theorem we need some preparation. At first we replace the space $S U_{X}(r, 0)$ by a suitable translate of it, namely the moduli space

$$
\mathcal{S}_{r}
$$

of semistable vector bundles $E$ on $X$ having rank $r$ and fixed determinant $\mathcal{O}_{X}(1)$ of degree $r(m+g-1)$. We assume that $X$ has general moduli and that $\mathcal{O}_{X}(1)$ is general in $\operatorname{Pic}^{r(m+g-1)}(X)$, with $m \geq 3$ and $r \geq 2$. In particular $\mathcal{O}_{X}(1)$ is very ample: we also assume that $X$ is embedded in $\mathbb{P}^{n}$ by $\mathcal{O}_{X}(1)$.

We recall that $\mathcal{S}_{r}$ is biregular to $S U_{X}(r, 0)$, the biregular map being induced by tensor product with an $r$-th root of $\mathcal{O}_{X}(-1)$.

Proposition 5.2. Let $E$ be a semistable vector bundle on $X$ with general moduli in $\mathcal{S}_{r}$. Then:

(i) $h^{0}(E)=r m$ and $\left(E, H^{0}(E)\right)$ is a good pair,

(ii) the Plücker form of $\left(E, H^{0}(E)\right)$ exists.

Proof. (i) It suffices to produce one semistable vector bundle $E$ on $X$, of degree $r(m+$ $g-1)$ and rank $r$, such that $h^{0}(E)=r m$ and $\left(E, H^{0}(E)\right)$ is a good pair in the sense of Definition 4.1. Then the statement follows because the conditions defining a good pair are open. Let $L \in \operatorname{Pic}^{m+g-1}(X)$ be general, then $h^{0}(L)=m$ and $L$ is globally generated. Since $m \geq 3, L$ defines a morphism birational onto its image

$$
f: X \rightarrow \mathbb{P}\left(H^{0}(L)^{*}\right) .
$$


Putting $E:=L^{\oplus r}$ we have a globally generated, semistable vector bundle such that $h^{0}(E)=r m$. Hence, to prove that $\left(E, H^{0}(E)\right)$ is a good pair, it remains to show that its classifying map

$$
g_{E}: X \rightarrow \mathbb{G}_{E}:=G\left(r, H^{0}(E)^{*}\right)
$$

is birational onto its image. We observe that $H^{0}(E)=H_{1} \oplus \cdots \oplus H_{r}$, where $H_{i}$ is just a copy of $H^{0}(L), i=1, \ldots, r$. Let $f_{i}: X \rightarrow \mathbb{P}\left(H_{i}^{*}\right)$ be the corresponding copy of $f$, for any $i=1, \cdots, r$. Then $g_{E}: X \rightarrow \mathbb{G}_{E}$ can be described as follows: let $\mathbb{P}\left(E_{x}^{*}\right) \subset \mathbb{P}\left(H^{0}(E)^{*}\right)$ be the linear embedding induced by the evaluation map, it turns out that $\mathbb{P}\left(E_{x}^{*}\right)$ is the linear span of $f_{1}(x), \ldots, f_{r}(x)$. This implies that $g_{E}=u \circ\left(f_{1} \times \cdots \times f_{r}\right)$, where

$$
u: \mathbb{P}\left(H_{1}^{*}\right) \times \cdots \times \mathbb{P}\left(H_{r}^{*}\right) \rightarrow \mathbb{G}_{E}
$$

is the rational map sending $\left(y_{1}, \ldots, y_{r}\right)$ to the linear span of the points $y_{i} \in \mathbb{P}\left(H_{i}^{*}\right) \subset$ $\mathbb{P}\left(H^{0}(E)^{*}\right), i=1, \ldots, r$. Since $f$ is birational onto its image, the same is true for the map $f_{1} \times \cdots \times f_{r}$. Moreover $u$ is clearly birational onto its image. Hence $g_{E}$ is birational onto its image. Finally $g_{E}$ is a morphism, since $L^{\oplus r}$ is globally generated. This completes the proof of (i).

(ii) Again it suffices to produce one good pair $\left(E, H^{0}(E)\right)$ with the required property. It is easy to see that this is the case if $E=L^{\oplus r}$ as in (i).

Now we consider the rational map

$$
\theta_{r, m}: \mathcal{S}_{r} \rightarrow\left|\mathcal{O}_{X}(1, \ldots, 1)\right|
$$

sending the moduli point $[E] \in \mathcal{S}_{r}$ of a general $E$ to the Plücker form

$$
\mathbb{D}_{E} \in\left|\mathcal{O}_{X}(1, \ldots, 1)\right|
$$

of the pair $\left(E, H^{0}(E)\right)$. Let $t \in \operatorname{Pic}^{m+g-1}(X)$ be an $r$-root of $\mathcal{O}_{X}(1)$, then we have a map

$$
a_{t}: X^{m} \rightarrow \operatorname{Pic}^{g-1}(X)
$$

sending $\left(x_{1}, \ldots, x_{m}\right)$ to $\mathcal{O}_{X}\left(t-x_{1}-\cdots-x_{m}\right)$. It is just the natural Abel map $a: X^{m} \rightarrow$ $\operatorname{Pic}^{m}(X)$, multiplied by -1 and composed with the tensor product by $t$. Fixing a Poincaré bundle $\mathcal{P}$ on $X \times \operatorname{Pic}^{g-1}(X)$ we have the sheaf

$$
R^{1} q_{2 *}\left(q_{1}^{*} E(-t) \otimes \mathcal{P}\right),
$$

where $q_{1}, q_{2}$ are the natural projection maps of $X \times \operatorname{Pic}^{g-1}(X)$. It is well known the support of this sheaf is either $\operatorname{Pic}^{g-1}(X)$ or a Cartier divisor $\Theta_{E}$, see [BNR]. Moreover, due to the choice of $t$, one has

$$
\Theta_{E} \in|r \Theta|,
$$

where $\Theta:=\left\{N \in \operatorname{Pic}^{g-1}(X) \mid h^{0}(N) \geq 1\right\}$ is the natural theta divisor of $\operatorname{Pic}^{g-1}(X)$. In particular, one has $h^{0}(E \otimes N(-t))=h^{1}(E \otimes N(-t))$ so that

$$
\text { Supp } \Theta_{E}=\left\{N \in \operatorname{Pic}^{g-1}(X) \mid h^{0}(E \otimes N(-t)) \geq 1\right\} .
$$

Finally, it is well known that there exists a suitable identification

$$
|r \Theta|=\mathbb{P}\left(H^{0}(\mathcal{L})^{*}\right)
$$

such that $\theta_{r}([E])=\Theta_{E},[\overline{B N R}]$. Computing Chern classes it follows

$$
a_{t}^{*} \Theta_{E}+r \Delta_{m} \in\left|\mathcal{O}_{X^{m}}(1, \ldots, 1)\right|,
$$


where $\Delta_{m} \subset X^{m}$ is the multidiagonal divisor. On the other hand, $r \Delta_{m}$ is a component of $\mathbb{D}_{E}$ by Proposition 4.8. Moreover, it follows from the definition of determinant divisor that $\mathbb{D}_{E}$ contains $a_{t}^{-1}\left(\Theta_{E}\right)$. Therefore we have

$$
a_{t}^{*} \Theta_{E}+r \Delta_{m}=\mathbb{D}_{E} .
$$

Let $\alpha:|r \Theta| \rightarrow\left|\mathcal{O}_{X^{m}}(1, \ldots, 1)\right|$ be the linear map sending $D \in|r \Theta|$ to $a_{t}^{*} D+r \Delta_{m}$. We conclude from the latter equality that

Proposition 5.3. $\theta_{r, m}$ factors through the theta map $\theta_{r}$, that is $\theta_{r, m}=\alpha \circ \theta_{r}$.

Proof of Theorem 5.1.

Let $\theta_{r, m}: \mathcal{S}_{r} \rightarrow\left|\mathcal{O}_{X^{m}}(1, \ldots, 1)\right|$ be as above. We have $\operatorname{Aut}(X)=1$ and $m \geq 3$. We know that $\left(E, H^{0}(E)\right)$ is a good pair if $[E] \in \mathcal{S}_{r}$ is general and that $\theta_{r, m}$ factors through the theta map $\theta_{r}$. Theorem 4.13 says that $\theta_{r, m}$ is generically injective if $\left(E, H^{0}(E)\right)$ is a good pair and the determinant map

$$
d_{E}: \wedge^{r} H^{0}(E) \rightarrow H^{0}\left(\mathcal{O}_{X}(1)\right)
$$

is injective for a general $[E]$. This is proved in the next section.

\section{THE INJECTIVITY OF THE DETERMINANT MAP}

Let $(X, E)$ be a pair such that $X$ is a smooth irreducible curve of genus $g$ and $E$ is a semistable vector bundle of rank $r$ on $X$ and degree $r(g-1+m)$, with $m \geq 3$. If $E$ is a general semistable vector bundle on $X$, it follows that:

(i) $\left(E, H^{0}(E)\right)$ is a good pair,

(ii) its Plücker form exists.

(see Definition 4.1 and Proposition 5.2). It is therefore clear that the previous conditions are satisfied on a dense open set $U$ of the moduli space of $(X, E)$.

Assumption: From now on we will assume that $(X, E)$ defines a point of $U$, so that $X$ is a general curve of genus $g$ and $E$ is semistable and satisfies (i) and (ii).

In this section we prove the following result:

Theorem 6.1. Let $X$ and $E$ be sufficiently general and $g>>r$, then:

(i) the determinant map $d_{E}: \wedge^{r} H^{0}(E) \rightarrow H^{0}(\operatorname{det} E)$ is injective,

(ii) the classifying map $g_{E}: X \rightarrow \mathbb{G}_{E}$ is an embedding.

Since $m \geq 3$, det $E:=\mathcal{O}_{X}(1)$ is very ample. So we will assume as usual that the curve $X$ is embedded in $\mathbb{P}^{n}=\mathbb{P}\left(H^{0}\left(\mathcal{O}_{X}(1)\right)^{*}\right)$. Let us also recall that

$$
\mathbb{G}_{E} \subset \mathbb{P}^{\left(\begin{array}{c}
r m \\
r
\end{array}\right)-1}
$$

denotes the Plücker embedding of the Grassmannian $G\left(r, H^{0}(E)^{*}\right)$. Let

$$
\lambda_{E}: \mathbb{P}^{n} \rightarrow \mathbb{P}^{\left(\begin{array}{r}
r m \\
r
\end{array}\right)-1}
$$

be the projectivized dual of $d_{E}$. We have already remarked in section 4 that $g_{E}$ is just the restriction $\lambda_{\left.E\right|_{X}}$. This immediately implies that

Lemma 6.2. $d_{E}$ is injective $\Leftrightarrow \lambda_{E}$ is surjective $\Leftrightarrow$ the curve $g_{E}(X)$ spans the Plücker space $\mathbb{P}\left(\begin{array}{c}r m \\ r\end{array}\right)-1$. 
Since $\left(E, H^{0}(E)\right)$ is a good pair, $g_{E}: X \rightarrow g_{E}(X)$ is a birational morphism. Let

$$
\left\langle g_{E}(X)\right\rangle \subset \mathbb{P}^{\left(\begin{array}{c}
r m \\
r
\end{array}\right)-1}
$$

be the linear span of $g_{E}(X)$. Then the previous Theorem 6.1 is an immediate consequence of the following one:

Theorem 6.3. For a general pair $(X, E)$ as above $g_{E}$ is an embedding and

$$
\operatorname{dim}\left\langle g_{E}(X)\right\rangle \geq r(m-1)+g .
$$

In other words, the statement says that $g_{E}$ is an embedding and that $d_{E}$ has rank $>$ $r(m-1)+g$. This theorem and the previous lemma imply that:

Corollary 6.4. For a general $(X, E), d_{E}$ is injective if $g \geq\left(\begin{array}{c}r m \\ r\end{array}\right)-r(m-1)-1$.

Hence the proof of Theorem 6.1 also follows.

Proof of Theorem 6.3.

To prove the theorem, hence Theorem 6.1, we observe that the moduli space of all pairs $(X, E)$ is an integral, quasi-projective variety defined over the moduli space $\mathcal{M}_{g}$ of $X$. On the other hand, the conditions in the statement of the theorem are open. Therefore, it suffices to construct one pair $(X, E)$ such that $E$ is semistable, $h^{0}(E)=r m$ and these conditions are satisfied. We will construct such a pair by induction on the genus

$$
g \geq 0
$$

of $X$. For $g=0$ we have $X=\mathbb{P}^{1}$ and $E=\mathcal{O}_{\mathbb{P}^{1}}(m-1)^{r}$.

Lemma 6.5. Let $X=\mathbb{P}^{1}$ and $E=\mathcal{O}_{\mathbb{P}^{1}}(m-1)^{r}$, with $m \geq 2$. Then $d_{E}$ is surjective and $g_{E}$ is an embedding.

Proof. The proof of the surjectivity of $d_{E}$ is standard. It also follows from the results in [T]. In order to deduce that $g_{E}$ is an embedding recall that $g_{E}$ is defined by Im $d_{E}$, hence by the complete linear system $\left|\mathcal{O}_{\mathbb{P}^{1}}(r(m-1))\right|$.

Now we assume by induction that the statement is true for $g$ and prove it for $g+1$.

Let $(X, E)$ be a general pair such that $X$ has genus $g$. We recall that then $X$ is a general curve of genus $g$ and $\left(E, H^{0}(E)\right)$ is a good pair admitting a Plücker form.

By induction $g_{E}$ is an embedding and $\operatorname{dim}\left\langle g_{E}(X)\right\rangle \geq r(m-1)+g$. We need to prove various lemmas.

Lemma 6.6. The evaluation map ev $v_{x, y}: H^{0}(E) \rightarrow E_{x} \oplus E_{y}$ is surjective for general $x, y \in X$.

Proof. If not we would have $h^{0}(E(-x-y))>h^{0}(E)-2 r=r(m-2)$, for any pair $(x, y) \in$ $X^{2}$. This implies that $h^{0}\left(E\left(-x-y-z_{1}-\cdots-z_{m-2}\right)\right) \geq 1, \forall\left(x, y, z_{1}, \ldots, z_{m-2}\right) \in X^{m}$ and hence that $\left(E, H^{0}(E)\right)$ has no Plücker form. But then, by Proposition 5.2 (ii), $(X, E)$ is not general: a contradiction.

From now on we put

$$
C:=g_{E}(X)
$$

Choosing $x, y$ so that $e v_{x, y}$ is surjective, we have a linear embedding

$$
E_{x}^{*} \oplus E_{y}^{*} \subset H^{0}(E)^{*}
$$


induced by the dual map $e v_{x, y}^{*}$. This induces an inclusion of Plücker spaces

$$
\mathbb{P}^{\left(\begin{array}{c}
2 r \\
r
\end{array}\right)-1}:=\mathbb{P}\left(\wedge^{r}\left(E_{x}^{*} \oplus E_{y}^{*}\right)\right) \subset \mathbb{P}^{\left(\begin{array}{c}
r m \\
r
\end{array}\right)-1}:=\mathbb{P}\left(\wedge^{r} H^{0}(E)^{*}\right)
$$

and of their corresponding Grassmannians

$$
\mathbb{G}_{x, y}:=G\left(r,\left(E_{x}^{*} \oplus E_{y}^{*}\right)\right) \subset \mathbb{G}_{E} .
$$

Lemma 6.7. Assume $\langle C\rangle$ is a proper subspace of the Plücker space of $\mathbb{G}_{E}$. Let $x, y$ be general points of $X$. Then $\left\langle\mathbb{G}_{x, y}\right\rangle$ is not in $\langle C\rangle$.

Proof. For a general $x \in X$ consider the linear map $\pi: H^{0}(E)^{*} \rightarrow H^{0}(E(-x))^{*}$ dual to the inclusion $H^{0}(E(-x)) \subset H^{0}(E)$. It induces a surjective linear projection

$$
\wedge^{r} \pi: \mathbb{P}\left(\wedge^{r} H^{0}(E)^{*}\right) \rightarrow \mathbb{P}\left(\wedge^{r} H^{0}(E(-x))^{*}\right),
$$

with center the linear span $\langle\sigma\rangle$ of $\sigma:=\left\{L \in \mathbb{G}_{E} \mid \operatorname{dim}\left(L \cap E_{x}^{*}\right) \geq 1\right\}$. In particular $\wedge^{r} \pi$ restricts to a rational map between Grassmannians

$$
f: \mathbb{G}_{E} \rightarrow \mathbb{G}_{E(-x)},
$$

where $\mathbb{G}_{E(-x)}:=G\left(r, H^{0}(E(-x))^{*}\right) \simeq G(r,(m-1) r)$. Let $l \in \mathbb{G}_{E}$ be the parameter point of the space $L$, then $f(l)$ is the parameter point of $\pi(L)$. Clearly $f$ is defined at $l$ iff $L \cap E_{x}^{*}=0$. Moreover, the closure of the fibre of $f$ at $f(l)$ is the Grassmannian $G\left(r, L \oplus E_{x}^{*}\right)$. In particular, the closure of the fibre at $f(y)$ is $\mathbb{G}_{x, y}$, for a general $y \in X$. We distinguish two cases:

(1) $f(C)$ spans the Plïcker space of $\mathbb{G}_{E(-x)}$. Since $f=\wedge^{r} \pi_{\left.\right|_{\mathbb{G}_{E}}}$ and $\wedge^{r} \pi$ is linear, it follows that $\bigcup_{y \in C}\left\langle\mathbb{G}_{x, y}\right\rangle$ spans the Plücker space of $\mathbb{G}_{E}$. Since $\langle C\rangle$ is proper in it, we conclude that $\left\langle\mathbb{G}_{x, y}\right\rangle$ is not in $\langle C\rangle$ for some $y$, hence for general points $x, y \in X$.

(2) $f(C)$ does not span the Plücker space of $\mathbb{G}_{E(-x)}$. Since the Plücker form of $\left(E, H^{0}(E)\right)$ exists and $m \geq 3$, we can fix $x, y, z_{1} \ldots z_{m-2} \in X$ so that $h^{0}(E(-x-y-z))=0$, where $z:=z_{1}+\cdots+z_{m-2}$. Then we have $H^{0}(E(-x)) \cap H^{0}(E(-y-z))=0$ in $H^{0}(E)$. Putting $E_{z}^{*}:=E_{z_{1}}^{*} \oplus \cdots \oplus E_{z_{i}}^{*}$, it follows that

$$
\pi_{\mid\left(E_{z}^{*} \oplus E_{y}^{*}\right)}: E_{y}^{*} \oplus E_{z}^{*} \rightarrow H^{0}(E(-x))^{*}
$$

is an isomorphism, that is, $\wedge^{r} \pi$ induces the following isomorphism of projective spaces:

$$
i_{y, z}: \mathbb{P}\left(\wedge^{r}\left(E_{y}^{*} \oplus E_{z}^{*}\right)\right) \rightarrow \mathbb{P}\left(\wedge^{r} H^{0}(E(-x))^{*}\right) .
$$

On the other hand, $\mathbb{P}\left(\wedge^{r}\left(E_{y}^{*} \oplus E_{z}^{*}\right)\right)$ is spanned by the union of its natural linear subspaces $\left\langle\mathbb{G}_{y, z_{i}}\right\rangle=\mathbb{P}\left(\wedge^{r}\left(E_{y}^{*} \oplus E_{z_{i}}^{*}\right)\right), i=1, . ., m-2$. Since $\langle f(C)\rangle$ is a proper subspace of $\mathbb{P}\left(\wedge^{r} H^{0}(E(-x))^{*}\right)$, it follows that $\left\langle\mathbb{G}_{y, z_{i}}\right\rangle$ is not in $\langle C\rangle$, for some $i=1, . ., m-2$.

Now we assume that $\langle C\rangle$ is a proper subspace of the Plücker space of $\mathbb{G}_{E}$ and fix general points $x, y \in X$ so that the conditions of the previous lemma are satisfied. Keeping the previous notations let $P \subset \mathbb{P}^{r m-1}$ be the tautological image of $\mathbb{P}\left(E^{*}\right)$ and let $P_{z}:=\mathbb{P}\left(E_{z}^{*}\right), z \in X$. We observe that the Grassmannian $\mathbb{G}_{x, y}$ is ruled by smooth rational normal curves of degree $r$ passing through $x$ and $y$. More precisely, let

$$
\mathbb{P}^{2 r-1}:=\mathbb{P}\left(E_{x}^{*} \oplus E_{y}^{*}\right)
$$

and for $t \in \mathbb{G}_{x, y}$ let

$$
P_{t} \subset \mathbb{P}^{2 r-1} \subset \mathbb{P}^{r m-1}
$$

be the projectivized space corresponding to $t$. We have: 
Lemma 6.8. For a general $t \in \mathbb{G}_{x, y}$ there exists a unique Segre product $S:=\mathbb{P}^{1} \times \mathbb{P}^{r-1}$ such that $P_{x} \cup P_{y} \cup P_{t} \subset S \subset \mathbb{P}^{2 r-1}$. Moreover:

(i) the ruling of $S$ is parametrized by a degree $r$ rational normal curve

$$
R \subset \mathbb{G}_{x, y} \subset \mathbb{G}_{E} \subset \mathbb{P}^{\left(\begin{array}{c}
r m \\
r
\end{array}\right)-1},
$$

(ii) the universal bundle $\mathcal{U}_{r}$ of $\mathbb{G}_{E}$ restricts to $\mathcal{O}_{\mathbb{P}^{1}}(-1)^{\oplus r}$ on $R$,

(iii) the restriction map $\left.H^{0}\left(\mathcal{U}^{*}\right) \rightarrow H^{0}\left(\mathcal{O}_{\mathbb{P}^{1}}(1)\right)^{\oplus r}\right)$ is surjective.

Proof. Since $x, y$ are general in $X$, Lemma 6.6 implies that $P_{x} \cap P_{y}=\emptyset$. Since $t$ is general in $\mathbb{G}_{x, y}$, we have $P_{t} \cap P_{x}=P_{t} \cap P_{y}=\emptyset$. It is a standard fact that the union of all lines in $\mathbb{P}^{2 r-1}$ meeting $P_{x}, P_{y}$ and $P_{t}$ is the Segre embedding $S \subset \mathbb{P}^{2 r-1}$ of the product $\mathbb{P}^{1} \times \mathbb{P}^{r-1}$, which is actually the unique Segre variety containing the above linear spaces, see [Ha1], p.26, 2.12. It is also well known that $S$ is the tautological image of the projective bundle associated to $\mathcal{O}_{\mathbb{P}^{1}}(1)^{\oplus r}$, see [Ha2]. Therefore, the map assigning to each point $p \in \mathbb{P}^{1}$ the fiber of $S$ over $p$ is the classifying map of $\mathcal{O}_{\mathbb{P}^{1}}(1)^{\oplus r}$. So it defines an embedding of $\mathbb{P}^{1}$ into the Grassmannian $\mathbb{G}_{x, y}$, whose image is a rational normal curve $R$. This implies (ii) and (iii).

Let $t \in \mathbb{G}_{x, y}$ be a sufficiently general point, where $x, y$ are general in $X$. Then, by Lemma 6.7, $t$ is not in the linear space $\langle C\rangle$. Since $\mathbb{G}_{x, y}$ is ruled by the family of curves $R$, we can also assume that $C \cup R$ is a nodal curve with exactly two nodes in $x$ and $y$. So far we have constructed a nodal curve

$$
\Gamma:=C \cup R
$$

such that

(i) $\Gamma$ has arithmetic genus $g+1$ and degree $r(m+g)$,

(ii) $\operatorname{dim}\langle\Gamma\rangle \geq \operatorname{dim}\langle C\rangle+1=r(m-1)+g+1$.

\section{Lemma 6.9.}

(i) The curve $\Gamma$ is smoothable in $\mathbb{G}_{E}$,

(ii) $h^{1}\left(\mathcal{O}_{\Gamma}(1)\right)=0$ and $h^{0}\left(\mathcal{O}_{\Gamma}(1)\right)=r(m+g)-g$.

Let $\mathcal{U}_{r}$ be the universal bundle on $\mathbb{G}_{E}$, we have also the vector bundle on $\Gamma$ :

$$
F:=\mathcal{U}_{r}^{*} \otimes \mathcal{O}_{\Gamma}
$$

\section{Lemma 6.10.}

(i) The restriction map $H^{0}\left(\mathcal{U}_{r}^{*}\right) \rightarrow H^{0}(F)$ is an isomorphism,

(ii) $h^{1}(F)=0$ and $h^{0}(F)=r m$.

Lemma 6.11. Let $x_{1}, \ldots, x_{m}$ be general points on $C$. Then $h^{0}\left(F\left(-x_{1}-\cdots-x_{m}\right)\right)=0$.

Proof. Let us recall that $C=g_{E}(X)$ and that $E \cong \mathcal{U}_{r}^{*} \otimes \mathcal{O}_{C}$. Under the assumptions made at the beginning of this section, $X$ is a general curve of genus $g,\left(E, H^{0}(E)\right)$ is a good pair admitting a Plücker form. This implies that $h^{0}\left(E\left(-x_{1}-\cdots-x_{m}\right)\right)=0$, where $x_{1}, \ldots, x_{m}$ are general points on $X$. Notice also that $F \otimes \mathcal{O}_{C} \cong E$ and that, by the previous lemma, the restriction map $H^{0}(F) \rightarrow H^{0}(E)$ is an isomorphism. .

Let $d:=x_{1}+\cdots+x_{m}$ and let $s \in H^{0}(F(-d))$. Then $s$ is zero on $X$ because $h^{0}(E(-d))=0$. In particular $s$ is zero on $\{x, y\}=C \cap R$. Hence its restriction on $R$ is a global section $s_{\mid R}$ of $\mathcal{O}_{R}(-x-y)$. But $F \otimes \mathcal{O}_{R}(-x-y)$ is $\mathcal{O}_{\mathbf{P}^{1}}(-1)^{\oplus r}$ so that $s_{\left.\right|_{R}}=0$. Hence $s$ is zero on $\Gamma$ and $h^{0}(F(-d))=0$. 
We are now able to complete the proof of Theorem 6.3, postponing the proofs of lemmas 6.9 and 6.10 .

Completion of the proof of Theorem 6.3:

We start from a curve $\Gamma=C \cup R$ as above. Therefore the component $C=g_{E}(X)$ is the embedding in $\mathbb{G}_{E}$ of a curve $X$ with general moduli and, by the previous lemma, there exists $\left(x_{1}, \ldots, x_{m}\right) \in C^{m}$ such that $h^{0}\left(F\left(-x_{1}-\cdots-x_{m}\right)\right)=0$. Now recall that, by lemma 6.9, the curve $\Gamma$ is smoothable in $\mathbb{G}_{E}$. This means that there exists a flat family

$$
\left\{X_{t}, t \in T\right\}
$$

of curves $X_{t} \subset \mathbb{G}_{E}$ such that: (1) $T$ is integral and smooth, (2) for a given $o \in T$ one has $X_{o}=\Gamma$, (3) $X_{t}$ is smooth for $t \neq o$. Let

$$
E_{t}:=\mathcal{U}_{r}^{*} \otimes \mathcal{O}_{X_{t}}
$$

For $t$ general we have $h^{1}\left(E_{t}\right)=h^{1}(F)=0$, by semicontinuity, and hence $h^{0}\left(E_{t}\right)=r m$. For the same reason, the determinant map $d_{t}: \wedge^{r} H^{0}\left(E_{t}\right) \rightarrow H^{0}\left(\mathcal{O}_{X_{t}}(1)\right)$ has rank bigger or equal to the rank of $d_{o}: \wedge^{r} H^{0}(F) \rightarrow H^{0}\left(\mathcal{O}_{\Gamma}(1)\right)$. This is equivalent to say that

$$
\operatorname{dim}\left\langle X_{t}\right\rangle \geq \operatorname{dim}\langle\Gamma\rangle \geq r(m-1)+g+1 .
$$

Then, for $t$ general, the pair $\left(X_{t}, E_{t}\right)$ satisfies the statement of Theorem 6.3.

To complete the proof of the theorem, it remains to show that $E_{t}$ is semistable for a general $t$. It is well known that $E_{t}$ is semistable if it admits theta divisor, see [B2]. This is equivalent to say that

$$
\Theta_{t}:=\left\{N \in \operatorname{Pic}^{m}\left(X_{t}\right) \mid h^{0}\left(E_{t} \otimes N^{-1}\right) \geq 1\right\} \neq \operatorname{Pic}^{m}\left(X_{t}\right),
$$

therefore $E_{t}$ is semistable if

$$
D_{t}:=\left\{\left(z_{1}, \ldots, z_{m}\right) \in X_{t}^{m} \mid h^{0}\left(E_{t}\left(-z_{1}-\cdots-z_{m}\right)\right) \geq 1\right\} \neq X_{t}^{m} .
$$

To prove that $D_{t} \neq X_{t}^{m}$ for a general $t$, we fix in $\mathbb{G}_{E}{ }^{m} \times T$ the family

$$
A:=\left\{\left(z_{1}, \ldots, z_{m} ; t\right) \in \mathbb{G}_{E}^{m} \times T \mid z_{1}, \ldots, z_{m} \in X_{t}-\operatorname{Sing}\left(X_{t}\right)\right\},
$$

which is integral and smooth over $T$. Then we consider its closed subset

$$
D:=\left\{\left(z_{1}, \ldots, z_{m} ; t\right) \in A \mid h^{0}\left(\mathcal{U}_{r}^{*} \otimes \mathcal{O}_{X_{t}}\left(-z_{1}-\cdots-z_{m}\right)\right) \geq 1\right\} .
$$

It suffices to show that $D$ is proper, so that $D_{t} \neq X_{t}^{m}$ for a general $t$. Since $E_{o}=F$, lemma 6.11 implies that $D \cap X_{o}^{m}$ is proper. Indeed there exists a point $\left(x_{1}, \ldots, x_{m}\right) \in$ $C^{m} \subset X_{o}^{m}$ so that $h^{0}\left(F\left(-x_{1}-\cdots-x_{m}\right)\right)=0$. Hence $D$ is proper.

Proof of Lemma 6.9.

(i) We will put $\mathbb{G}:=\mathbb{G}_{E}$. We recall that $\Gamma$ is smoothable in $\mathbb{G}$ if there exists an integral variety $\mathcal{X} \subset \mathbb{G} \times T$ such that:

(a) the projection $p: \mathcal{X} \rightarrow T$ is flat,

(b) for some $o \in T$ the fibre $\mathcal{X}_{o}$ is $\Gamma$,

(c) if $t \in T-\{o\}$, the fibre $\mathcal{X}_{t}$ is smooth of genus $g+1$. 
To prove that $\Gamma$ is smoothable we use a well known argument, see $[\mathrm{S}]$ or $[\mathrm{HH}]$. Consider the natural map $\phi: \mathcal{T}_{\mathbb{G} \mid \Gamma} \rightarrow \mathcal{N}_{\Gamma \mid \mathbb{G}}$, where $\mathcal{N}_{\Gamma \mid \mathbb{G}}$ is the normal bundle of $\Gamma$ in $\mathbb{G}$. The Cokernel of $\phi$ is a sheaf $T_{S}^{1}$, supported on $S:=\operatorname{Sing}(\Gamma)$. It is known as the $T^{1}$-sheaf of Lichtenbaum-Schlessinger. Finally, $\phi$ fits into the following exact sequence induced by the inclusion $\Gamma \subset \mathbb{G}$ :

$$
0 \rightarrow \mathcal{T}_{\Gamma} \rightarrow \mathcal{T}_{\mathbb{G} \mid \Gamma} \stackrel{\phi}{\rightarrow} \mathcal{N}_{\Gamma \mid \mathbb{G}} \rightarrow T_{S}^{1} \rightarrow 0
$$

Let $\mathcal{N}^{\prime}$ be the image of $\phi$ in $\mathcal{N}_{\Gamma \mid \mathbb{G}}$. The condition $h^{1}\left(\mathcal{N}^{\prime}\right)=0$ implies that $\Gamma$ is smoothable in $\mathbb{G},[S]$ prop. 1.6. To show that $h^{1}\left(\mathcal{N}^{\prime}\right)=0$ it is enough to show that $h^{1}\left(\mathcal{T}_{\mathbb{G} \mid \Gamma}\right)=0$, this is a standard argument following from the exact sequence

$$
0 \rightarrow \mathcal{T}_{\Gamma} \rightarrow \mathcal{T}_{\mathbb{G} \mid \Gamma} \rightarrow \mathcal{N}^{\prime} \rightarrow 0
$$

To prove that $h^{1}\left(\mathcal{T}_{\mathbb{G} \mid \Gamma}\right)=0$ we use the Mayer-Vietoris exact sequence

$$
0 \rightarrow \mathcal{T}_{\mathbb{G} \mid \Gamma} \rightarrow \mathcal{T}_{\mathbb{G} \mid C} \oplus \mathcal{T}_{\mathbb{G} \mid R} \rightarrow \mathcal{T}_{\mathbb{G} \mid S} \rightarrow 0
$$

The associated long exact yields the restriction map

$$
\rho: H^{0}\left(\mathcal{T}_{\mathbb{G} \mid C}\right) \oplus H^{0}\left(\mathcal{T}_{\mathbb{G} \mid R}\right) \rightarrow H^{0}\left(\mathcal{T}_{\mathbb{G} \mid S}\right) .
$$

At first we show its surjectivity: it suffices to show that

$$
\rho: 0 \oplus H^{0}\left(\mathcal{T}_{\mathbb{G} \mid R}\right) \rightarrow H^{0}\left(\mathcal{T}_{\mathbb{G} \mid S}\right)
$$

is surjective. Recall that $S$ consists of two points $x, y$ and that $T_{S}^{1}=\mathcal{O}_{S}$. Then, tensoring by $\mathcal{T}_{\mathbb{G} \mid R}$ the exact sequence

$$
0 \rightarrow \mathcal{O}_{R}(-x-y) \rightarrow \mathcal{O}_{R} \rightarrow \mathcal{O}_{S} \rightarrow 0
$$

the surjectivity of $\rho$ follows if $h^{1}\left(\mathcal{T}_{\mathbb{G} \mid R}(-x-y)\right)=0$. To prove this consider the standard Euler sequence defining the tangent bundle to $\mathbb{G}$ :

$$
0 \rightarrow \mathcal{U}_{r} \otimes \mathcal{U}_{r}^{*} \rightarrow O_{\mathbb{G}}^{\oplus r m} \otimes \mathcal{U}_{r}^{*} \rightarrow \mathcal{T}_{\mathbb{G}} \rightarrow 0
$$

Then restrict it to $R$ and tensor by $\mathcal{O}_{R}(-x-y)$. The term in the middle of such a sequence is $M:=\mathcal{O}_{\mathbb{P}^{1}}^{\oplus r m} \otimes \mathcal{O}_{\mathbb{P}^{1}}(-1)^{\oplus r}$. This just follows because $\mathcal{U}_{r}^{*} \otimes \mathcal{O}_{R} \cong \mathcal{O}_{\mathbb{P}^{1}}(1)^{\oplus r}$. Since $h^{1}(M)=0$, it follows that $h^{1}\left(\mathcal{T}_{\mathbb{G} \mid R}(-x-y)\right)=0$. Hence $\rho$ is surjective. The surjectivity of $\rho$ and the vanishing of $h^{1}\left(\mathcal{T}_{\mathbb{G} \mid R}\right)$ and $h^{1}\left(\mathcal{T}_{\mathbb{G} \mid C}\right)$ clearly imply that $h^{1}\left(\mathcal{T}_{\mathbb{G} \mid \Gamma}\right)=0$. Hence we are left to show that $h^{1}\left(\mathcal{T}_{\mathbb{G} \mid R}\right)=h^{1}\left(\mathcal{T}_{\mathbb{G} \mid C}\right)=0$. Since $\mathcal{T}_{\mathbb{G} \mid R} \cong \mathcal{O}_{\mathbb{P}^{1}}^{\oplus r m} \otimes \mathcal{O}_{\mathbb{P}^{1}}(1)^{\oplus r}$, the former vanishing is immediate. To prove that $h^{1}\left(\mathcal{T}_{\mathbb{G} \mid C}\right)=0$ the argument is similar. Restricting the above Euler sequence to $C$ we obtain the exact sequence

$$
0 \rightarrow E^{*} \otimes E \rightarrow E^{\oplus r m} \rightarrow \mathcal{T}_{\mathbb{G} \mid C} \rightarrow 0,
$$

since $\mathcal{U}_{r \mid C}^{*} \simeq E$. Then $h^{1}(E)=0$ implies $h^{1}\left(\mathcal{T}_{\mathbb{G} \mid C}\right)=0$.

(ii) To prove $h^{1}\left(\mathcal{O}_{\Gamma}(1)\right)=0$ it suffices to consider the long exact sequence associated to the Mayer-Vietoris exact sequence

$$
0 \rightarrow \mathcal{O}_{\Gamma}(1) \rightarrow \mathcal{O}_{C}(1) \oplus \mathcal{O}_{R}(1) \rightarrow \mathcal{O}_{x, y}(1) \rightarrow 0 .
$$

For degree reasons we have $h^{1}\left(\mathcal{O}_{C}(1)\right)=h^{1}\left(\mathcal{O}_{R}(1)\right)=0$. Hence it suffices to show that the restriction $H^{0}\left(\mathcal{O}_{C}(1)\right) \oplus H^{0}\left(\mathcal{O}_{R}(1)\right) \rightarrow \mathcal{O}_{x, y}$ is surjective. This follows from the surjectivity of the restriction $H^{0}\left(\mathcal{O}_{R}(1)\right) \rightarrow \mathcal{O}_{x, y}$. 
Proof of Lemma 6.10.

Tensoring by $F$ the standard Mayer-Vietoris exact sequence

$$
0 \rightarrow \mathcal{O}_{\Gamma} \rightarrow \mathcal{O}_{C} \oplus \mathcal{O}_{R} \rightarrow \mathcal{O}_{x, y} \rightarrow 0
$$

we have the exact sequence

$$
0 \rightarrow F \rightarrow E \oplus \mathcal{O}_{\mathbb{P}^{1}}(1)^{\oplus r} \rightarrow F \otimes \mathcal{O}_{x, y} \rightarrow 0 .
$$

Passing to the associated long exact sequence we obtain

$$
0 \rightarrow H^{0}(F) \stackrel{u}{\rightarrow} H^{0}(E) \oplus H^{0}\left(\mathcal{O}_{\mathbb{P}^{1}}(1)^{\oplus r}\right) \stackrel{\rho}{\rightarrow} H^{0}\left(F \otimes \mathcal{O}_{x, y}\right) \rightarrow H^{1}(F) \rightarrow 0
$$

Restricting $\rho$ to $H^{0}(E) \oplus 0$ or $0 \oplus H^{0}\left(\mathcal{O}_{\mathbb{P}^{1}}(1)^{\oplus r}\right)$ we have the following maps

$$
\rho_{C}: H^{0}(E) \rightarrow E_{x} \oplus E_{y},
$$

and

$$
\rho_{R}: H^{0}\left(\mathcal{O}_{\mathbb{P}^{1}}(1)^{\oplus r}\right) \rightarrow \mathcal{O}_{\mathbb{P}^{1}, x}(1)^{\oplus r} \oplus \mathcal{O}_{\mathbb{P}^{1}, y}(1)^{\oplus r}
$$

These are the usual evaluation maps and we know they are surjective. It follows from the surjectivity of $\rho$ and the above long exact sequece that $h^{0}(F)=r m=h^{0}\left(\mathcal{U}_{r}^{*}\right)$ and $h^{1}(F)=0$. Thus, to complete the proof, it suffices to show that $H^{0}\left(\mathcal{U}_{r}^{*}\right) \rightarrow H^{0}(F)$ is injective. This is clear because the composition of maps $H^{0}\left(\mathcal{U}_{r}^{*}\right) \rightarrow H^{0}(F) \rightarrow H^{0}(E)$ is injective.

\section{REFERENCES}

[B1] A. Beauville, Fibrés de rang 2 sur les courbes, fibré detérminant et fonctions theta, Bull. Soc. Math. France, 116 (1988), 431-448.

[B2] A. Beauville, Vector bundles on curves and theta functions, in "Moduli Spaces and Arithmetic Geometry" (Kyoto 2004), Advanced Studies in pure Math. 45, 2006, 145-156.

[B3] A. Beauville, Vector bundles and theta functions on curves of genus 2 and 3, Amer.J. of Math. 128 (2006), 607-618.

[BNR] A. Beauville, M.S. Narasimhan, S. Ramanan, Spectral curves and the generalised theta divisor, J.Reine Angew. Math. 398, 169-179 (1989).

[BV1] S.Brivio and A. Verra, The theta divisor of $S U_{C}(2,2 d)^{s}$ is very ample if $C$ is not hyperelliptic, Duke Math.J., 82(1996), 503-552.

[BV2] S.Brivio and A. Verra, The Brill Noether curve of a stable vector bundle on a genus two curve, in "Algebraic Cycles and Motives", London Mathematical Society, LNS N 344, vol 2 (2007), Editors Jan Nagel and Chris Peters, Cambridge University Press.

[CGT] S. Casalaina-Martin, T. Gwena and M. Teixidor i Bigas, Some examples of vector bundles in the base locus of the generalized theta divisor, C. R. Acad. Sci. Paris Ser. I. Math., 347 (2009).

[D] I.Dolgachev Topics in classical Algebraic Geometry http:/ / www.math.lsa.umich.edu/ idolga/lecturenotes.html (2005)

[DN] J. M. Drézet and M.S. Narasimhan, Groupe de Picard des variétés de modules de fibrés semi-stable sur les courbes algébriques, Invent. Math. 97 (1989) 53-94

[DR] U.V. Desale and S. Ramanan Classification of vector bundles of rank 2 on hyperelliptic curves Invent. math. 38 (1976) 161-185

[G] W.H.Greub, Multilinear Algebra, Springer Verlag, Berlin Geidelberg New York 1967.

[Ha1] J. Harris, Algebraic Geometry - A first Course, Springer-Verlag New York, 1992.

[Ha2] J. Harris, A bound on the geometric genus of projective varieties, Ann. Scuola Norm. Sup. Pisa, (4), 8 , (1981) 35-68.

[H] A. Hirschowitz, Problemes de Brill Noether en rang superior, C. R. Acad. Sci. Paris Ser I Math. 307, (1988), 153-156.

[HH] A. Hirschowitz and R. Hartshorne, Smoothing algebraic space curves Lecture Notes in Math. 1124 (1985) 98-131 
[L] J.Le Potier, Faisceaux semi-stables and systemes coherents, in Vector bundles in Algebraic Geometry, Durham 1993, edit. N.J.Hitchin, P.E. Newstead and W.M. Oxbury, LMS Lectures Notes Series 208, Cambridge University Press (1995), pp.179-239.

[Pa] C. Pauly, Rank four vector bundles without theta divisor over a curve of genus two to appear in Advances in Geom. (2009)

[R] M. Raynaud, Sectiones des fibrés vectoriels sur une courbe, Bull. Soc. Math. France 110 (1982), 103-125.

[S] E. Sernesi, On the existence of certain families of curves, Invent. Math. 75, (1984), 25-57.

[T] M.Teixidor I Bigas, Curves in Grassmannian, Proc. Amer. Math. Soc. 126 (1998), 1597-1603.

[vGI] B. van Geemen and E. Izadi The tangent space to the moduli space of vector bundles on a curve and the singular locus of the theta divisor of the Jacobian, J. Alg. Geom. 10 (2001) 133-177

Universitá di Pavia, Dipartimento di Matematica, strada Ferrata 1 Pavia; email: SoNIA.BRIVIO@UNIPV.IT

Universitá Roma Tre, Dipartimento di Matematica, L.go S. Leonardo Murialdo, 1 00146 ROMA; EMAIL: VERRA@MAT.UNIROMA3.IT 